\title{
Microscopic Theory of a System of Interacting Bosons-I : Basic Foundations and Superfluidity
}

\author{
Yatendra S. Jain
}

Department of Physics, North-Eastern Hill University, Shillong, 793022, India

\begin{abstract}
Important aspects of a system of interacting bosons like liquid ${ }^{4} \mathrm{He}$ are critically analyzed to lay down the basic foundations of a new approach to develop its microscopic theory that explains its properties at quantitative level. It is shown that each particle represents a pair of particles (identified as the basic unit of the system) having equal and opposite momenta $(\mathbf{q},-\mathbf{q})$ with respect to their center of mass $(\mathrm{CM})$ that moves as a free particle with momentum $\mathbf{K}$; its quantum state is represented by a macro-orbital which ascribes a particle to have two motions ( $q$ and $K$ ) of the representative pair. While $q$ is restricted to satisfy $q \geq q_{\mathrm{o}}=\pi / d$ ( $d$ being the nearest neighbor distance) due to hard core inter-particle interaction, $K$, having no such restriction, can have any value between 0 and $\infty$. In the ground state of the system, all particles have: (i) $q=q_{\mathrm{o}}$ and $K=0$, (ii) identically equal nearest neighbor distance $r(=d)$, and (iii) relative phase positions locked at $\Delta \varphi(=2 \mathbf{q} \cdot \mathbf{r})=2 n \pi(n=1,2$, $3 .$. ); they define a close packed arrangement of their wave packets (CPA-WP) having identically equal size, $\lambda / 2=d$. The transition to superfluid state represents simultaneous onset of Bose Einstein condensation of particles in the state of $q=q_{\mathrm{o}}$ and $K=0$ and an order-disorder process which moves particles from their disordered positions in phase space (with $\Delta \varphi \geq 2 \pi$ in the high temperature phase) to an ordered positions defined by $\Delta \varphi=2 n \pi$ (in the low temperature phase). Quantum correlation potentials play an important role in this process. Particles in the superfluid state cease to have relative motion. They develop a kind of collective binding energy $\left(E_{g}(T)\right)$, identified as an energy gap between normal liquid state and superfluid state. These inferences help in understanding all significant properties of the superfluid state including loss of viscosity, quantized vortices, critical velocities, infinitely high thermal conductivity, thermo-mechanical and mechano-caloric effects, etc. at quantitative scale; however, this fact is demonstrated in detail, elsewhere.
\end{abstract}

Keywords Superfluidity, Microscopic theory, BEC, He-4

\section{Introduction}

Liquid ${ }^{4} \mathrm{He}$ (LHe-4)[a system of interacting bosons (SIB)] exhibits an interesting and extensively investigated phase transition at $T_{\lambda}=2.17 \mathrm{~K}[1-5]$. While its high temperature (HT) phase (He-I) has properties of a normal liquid, the low temperature (LT) phase (He-II) assumes several unique properties such as superfluidity, -flow through narrow channels without resistance (i.e., viscosity, $\eta=0$ ). For the last seventy five years, superfluidity of He-II has been in focus for its widely different experimental and theoretical studies because it serves as a testing ground for the impacts of the wave nature of particles at macroscopic level and this has been reflected by a large number of research papers on the related physics published every year.

The observation of superfluidity of LHe-4[1] in 1938, gave great boost to its experimental and theoretical studies. Within a short period of about three years enough

* Corresponding author:

profysjain@gmail.com (Yatendra S. Jain)

Published online at http://journal.sapub.org/ajcmp

Copyright (C) 2012 Scientific \& Academic Publishing. All Rights Reserved experimental results were available[3] for a phenomenological theory developed by Landau[6] which identified He-II as a homogeneous mixture of two fluids, i.e., superfluid (density $\rho_{\mathrm{s}}$ ) and normal fluid (density $\rho_{\mathrm{n}}=\rho-\rho_{\mathrm{s}}$ with $\rho=$ total density of He-II) of different properties[7]; the theory is well known as Landau's two fluid theory and it is elegantly discussed by Putterman[5]. Similarly, after a few more years Bogoliubov[8] reported his theory of a system of weakly interacting bosons as a most significant contribution towards the development of the microscopic theory of a SIB and concluded that the density of Bose Einstein condensate (BEC) (also known as zero momentum $(p=0)$ condensate $[9,10])$ is depleted if the bosons have repulsive interaction and the depletion increases with the increase in its strength; this[8] rendered a reason to believe in London's suggestion[11] that superfluidity of He-II originates from the existence of $p=0$ condensate of ${ }^{4} \mathrm{He}$-atoms which was presumed to be an important basis for further developments in the theory of a SIB.

London's suggestion[11] was further strengthened by a series of papers by Feynman and his collaborators[12-16] published during 1952-58. Starting from the exact quantum mechanical partition function of LHe-4, Feynman[12] not only showed that: (i) strong repulsion between ${ }^{4} \mathrm{He}$-atoms 
does not change $\lambda$-transition qualitatively from BE- condensation, and (ii) the spectrum of the elementary excitations of He-II (as perceived by Landau[6] to develop his two fluid theory) has quantum mechanical basis[13-16] but also discovered the origin of quantum vortices and their relation with criticality of He-II for its flow under rotation and through narrow channels[15]. In addition, Cohen and Feynman[17] predicted that the amplitude of neutron inelastic scattering (NIS) from quasi-particle excitations of LHe-4 should be reasonably large and this was later proved to be true through experiment[4]. In a nut shell, these studies[12-17] made Landau's two fluid theory[6] more meaningful because its phenomenological concepts of phonons and rotons, which determine $\rho_{\mathrm{n}}(T)$ and $\rho_{\mathrm{s}}(T)$, were shown to have microscopic foundation.

In what follows after[8] and[12-16], while the main objective of the theoretical studies of LHe-4 remained to: (i) formulate the relation for its excitation spectrum, $E(Q)$, and (ii) calculate the amount of $p=0$ condensate (i.e., the fraction of the total number of particles $(N)$ in $p=0$ state $n_{\mathrm{p}=0}(T)$ $\left.=N_{\mathrm{p}=0}(T) / N\right)$ in He-II, the experimental studies were aimed at: (i) finding the details of $E(Q)$, (ii) confirming the presumed existence of $p=0$ condensate and finding its magnitude and (iii) revealing other aspects of significance such as quantum vortices. To this effect, while Bogoliubov's theory[8] emerged as the starting point for the development of the microscopic theory of a SIB by using perturbative approach and second quantization[18], Feynman's work[12-16] laid the foundation for the application of variational approach of quantum theory[19] and path integrals to calculate different properties of a many body quantum system (MBQS). This motivated a large number of researchers to undertake theoretical study of LHe-4 by using different possible presumptions, approximations, trial functions, mathematical tools and computational techniques and encouraged many experimentalists to measure its different properties. The wealth of theoretical and experimental results, so generated, have been periodically reviewed and analyzed in large number of books and review articles. Here we refer some of them for their relevance to the present study. These are broadly related to: (i) the progress of microscopic understanding of a SIB[20-23], (ii) computer simulations of the properties of LHe-4[24], (iii) the values of $n_{\mathrm{p}=0}(T)$ in He-II[25-28], (iv) $E(Q)$ of LHe-4[29-31], and (v) quantum vortices[32, 33]. The basic understanding of the subject, so developed, is also summarized elegantly in graduate level texts[34-36]. In what follows, the unique properties of He-II are understood in terms of two fluid theory[6] and/or $\Psi$-theory[37] with a touch of microscopic basis which correlates $\rho_{\mathrm{s}}(T)$ with $n_{\mathrm{p}=0}(T)$ which is estimated to fall around $10 \%$ for He-II at $T$ $=0[28]$. However, this conclusion not only faces several unanswered questions[38] but also finds no unequivocal experimental support for the existence of the presumed $p=0$ condensate in He-II, -as evident from a discussion by Glyde and Svensson[25], Sokol[28] and Leggett[39].

Several research groups have been trying to revise our understanding of the superfluid state of a SIB, particularly, after the discovery of BEC in trapped dilute gases (TDG) and their detailed studies[40-42]. While some researchers[43-46] have been trying to establish the idea that the said state has a kind of generalized/composite condensate ( $p=0$ condensate + pair condensate + ..), others[47, 48] have been making their efforts to underline the condensation of Cooper type pairs only. Developments in these directions are reviewed, recently, in several articles, viz.,[49-51].

As such, it is evident that the real form of condensate responsible for the superfluidity of a SIB is unclear and a microscopic theory which explains the properties of LHe-4 at quantitative scale is still awaited. Guided by this observation we worked out a new approach to develop our non-conventional microscopic theory (NCMT) of a SIB by solving the $\mathrm{N}$ particle differential Schrodinger equation. We note that different approaches (viz., variational quantum approach and second quantization clubbed with perturbation techniques) to conventional microscopic theory (CMT) of a SIB use single particle basis (SPB) in a sense that each particle is presumed to represent a single particle kept in a box of volume $\mathrm{V}$ of the system with its states described by plane waves; in other words a single particle represents the basic unit of the system. However, for the reasons which become clear in the process of the formulation of our theory, we use pair of particles basis (PPB) which identifies a pair of particles as the basic unit of the system.

The paper is arranged as follows. The Hamiltonian of a SIB like LHe-4 and the wave mechanics of a pair of particles are critically analyzed to lay down the basic foundations of our approach in Section 2. While the wave function of $\mathrm{N}$-particle states (with corresponding eigen energies) and ground state (G-state) configuration are determined in Section 3 , a detailed analysis of the $\lambda$-transition and related aspects is studied in Section 4. The origin of bound pair formation and energy gap (a kind of collective binding among all the $N$ particles) between superfluid (S) and normal fluid $(\mathrm{N})$ phases of the system and its relationship with superfluidity and related aspects are revealed in Section 5 . Important aspects of Bogoliubov's picture, two fluid theory, $\Psi$-theory, etc. and crucial experimental observations which render decisive support to our theory are briefly analyzed in Section 6. While important aspects of our theory and CMT are summarized in Section 7 for their comparison, useful concluding remarks are made in Section 8.

\section{Basic aspects of a SIB}

\subsection{Hamiltonian}

An N particle SIB like LHe-4 is described[9], to a good approximation, by

$$
H(N)=\Sigma_{i}^{N} h_{i}+\Sigma_{\mathrm{i}>\mathrm{j}} V\left(r_{i j}\right) ; \text { with } h_{i}=-\left(\hbar^{2} / 2 m\right) \nabla_{i}^{2}
$$

where $m$ is the mass of a particle and $V\left(r_{i j}\right)=V^{\mathrm{R}}\left(r_{i j}\right)+V^{\mathrm{A}}\left(r_{i j}\right)$ is a two body central force potential with (i) $V^{R}\left(r_{i j}\right)$, being the short range strong repulsion and (ii) $V^{\mathrm{A}}\left(r_{i j}\right)$, a weak attraction of slightly longer range. While $V^{R}\left(r_{i j}\right)$ can be approximated to hard core $(\mathrm{HC})$ interaction $V_{\mathrm{HC}}\left(r_{i j}\right)$ [defined by $V_{\mathrm{HC}}\left(r_{i j} \leq \sigma\right)=$ 
$\infty$ and $V_{\mathrm{HC}}\left(r_{i j}>\sigma\right)=0$ with $\sigma$ being the $\mathrm{HC}$ diameter of a particle], $V^{\mathrm{A}}\left(r_{i j}\right)$ renders a flat surface of constant -ve potential (say, $-V_{\mathrm{o}}$ ) for the particles (hard spheres) to remain confined to the volume $(\mathrm{V})$ of the liquid and to move freely unless they collide with each other or the boundaries of the system (the free surface of the liquid and walls of the container). As discussed in Section 5.1, $V^{\mathrm{A}}\left(r_{i j}\right)$ also serves as a source of collective binding of all the $N$ particles in the S-phase of a SIB. Foregoing $-V_{\mathrm{o}}$ for its constant value, the effective Hamiltonian of LHe-4 can be written as,

where we use

$$
H_{\mathrm{o}}(N)=\Sigma_{\mathrm{i}}^{N} h_{i}+\Sigma_{\mathrm{i}>\mathrm{j}} \mathrm{A}\left(r_{i j}\right) \delta\left(r_{i j}\right)
$$

$$
V_{\mathrm{HC}}\left(r_{i j}\right) \equiv \mathrm{A}\left(r_{i j}\right) \delta\left(r_{i j}\right)
$$

with $\mathrm{A}\left(r_{i j}\right)$ being the strength of Dirac delta $\delta\left(r_{i j}\right)$ repulsion. For impenetrable $\mathrm{HC}$ particles, we have $\mathrm{A}\left(r_{i j}\right) \rightarrow \infty$ with $r_{i j}$ $\rightarrow 0$.

With a view to find the physical basis of Eqn.3 which agrees closely with a similar relation derived mathematically by Huang[52], we examine the possible configuration of two $\mathrm{HC}$ particles (say, $\mathrm{P} 1$ and $\mathrm{P} 2$ ) right at the instant of their collision which occurs with $r_{C M}(1)=\sigma / 2$ and $r_{C M}(2)=-\sigma / 2$ where $r_{C M}(i=1$ or 2) represents the distance of the center of mass (CM) of $i$-th particle from the $\mathrm{CM}$ of the pair (i.e., P1 and $\mathrm{P} 2)$. We note that $\mathrm{P} 1$ and $\mathrm{P} 2$ encounter $V_{\mathrm{HC}}\left(r_{i j}\right)$ only when they register their physical touch at the instant of their collision; no force can compress them beyond this touch. The process of collision does not register how far are the $\mathrm{CM}$ points of individual $\mathrm{P} 1$ and $\mathrm{P} 2$ from the point of this touch which is not different from the $\mathrm{CM}$ of the pair. The change in potential energy of P1 and P2 during their collision is, obviously, independent of their $\sigma$ and this justifies Eqn.3. We understand that this equivalence would not be justified in relation to certain physical aspects of a SIB (e.g., the volume occupied by a given number of particles) where the real size of the particle assumes importance.

\subsection{Dynamics of a Pair of Particles}

Although, particles in a SIB like LHe-4 under certain physical conditions can be treated, to a good approximation, as independent particles, even CMTs based on SPB consider particles in pairs to evaluate their potential energy since they interact through two body interactions, $V\left(r_{i j}\right)$. This shows that consideration of single particle as a basic unit of the system does not have general validity. Evidently, for a complete and better understanding of a SIB we need to consider a pair of particles as its basic unit whose dynamics can be described in the CM coordinate system by

$$
H_{0}(2) \Psi(r, R)=\left[\left(\hbar^{2} / 4 m\right)\left(-\nabla_{R}^{2}-4 \nabla r^{2}\right)+\mathrm{A}\left(r_{i j}\right) \delta\left(r_{i j}\right)\right] \Psi(r, R)=
$$

$$
E(2) \Psi(r, R)
$$

with

$$
\Psi(r, R)=\psi_{\mathrm{k}}(\mathbf{r}) \exp (i \mathbf{K} . \mathbf{R})
$$

where

$$
\mathbf{k}=\mathbf{p}_{2}-\mathbf{p}_{1}=2 \mathbf{q} \text { and } \mathbf{K}=\mathbf{p}_{2}+\mathbf{p}_{1}
$$

respectively, define their relative and CM momenta, and

$$
\mathbf{r}=\mathbf{b}_{2}-\mathbf{b}_{1} \text {, and } \mathbf{R}=\mathbf{b}_{2}+\mathbf{b}_{1}
$$

define their relative and CM positions with $\mathbf{b}_{1}$ and $\mathbf{b}_{2}$ being their positions and $\mathbf{p}_{1}$ and $\mathbf{p}_{2}$ being their momenta (in wave number).

In order to find $\Psi(r, R)$ (Eqns.4 and 5) we may treat $\mathrm{A}(r) \delta(r)$ as a step potential, since it has infinitely large $+v e$ value only at $r=0$. Consequently, the quantum state of P1 and $\mathrm{P} 2$ at all points (excluding $r=0$ ) can be represented, to a good approximation, by with

$$
\Psi(1,2)^{ \pm}=(1 / \sqrt{ } 2)\left[u_{\mathbf{p} 1}\left(\mathbf{b}_{1}\right) \mathrm{u}_{\mathbf{p} 2}\left(\mathbf{b}_{2}\right) \pm \mathrm{u}_{\mathbf{p} 2}\left(\mathbf{b}_{1}\right) \mathrm{u}_{\mathbf{p} 1}\left(\mathbf{b}_{2}\right)\right]
$$

$$
\mathrm{u}_{\mathrm{pi}}\left(\mathbf{b}_{\mathrm{i}}\right)=\exp \left(i \mathbf{p}_{i} \cdot \mathbf{b}_{i}\right)
$$

being a plane wave having unit normalization. However, for the fact $\mathrm{A}(r) \delta(r)=\infty$ at $r=0$, we use the condition that $\Psi(1,2)^{ \pm}=0$ at $r=0$. Rearranging $\Psi(1,2)^{ \pm}$(Eqn.8) in terms of CM coordinates, we have

$$
\Psi(r, R)^{ \pm}=\sqrt{ } 2 \psi_{\mathbf{k}}(\mathbf{r})^{ \pm} \exp (\mathrm{iK} . \mathbf{R})
$$

with

$$
\psi_{\mathbf{k}}(\mathbf{r})^{-}=\sin (\mathbf{q} \cdot \mathbf{r}) \text { and } \psi_{\mathbf{k}}(\mathbf{r})^{+}=\cos (\mathbf{q} \cdot \mathbf{r})
$$

by using $\mathbf{k}=2 \mathbf{q}$. Here we note that $\Psi(r, R)^{-}$(Eqn.10), satisfying $\left.\Psi(r, R)\right|_{r=0}=0$, has $-v e$ symmetry for the exchange of $\mathrm{P} 1$ and $\mathrm{P} 2$ for which it can be an acceptable wave function for two fermions (not for two bosons) but $\Psi(1,2)^{+}$(Eqn.10), having desired $+v e$ symmetry for bosons, does not satisfy $\left.\Psi(r, R)^{+}\right|_{r=0}=0$. Consequently, we need an alternative of $\Psi(r, R)^{+}$to describe a pair of $\mathrm{HC}$ bosons and we find

$\Phi(r, R)^{+}=\sqrt{ } 2 \phi_{\mathbf{k}}(\mathbf{r})^{+} \exp (\mathbf{i K} . \mathbf{R})$ with $\varphi_{\mathbf{k}}(\mathbf{r})^{+}=\sin |(\mathbf{q . r})|(12)$

which not only has $+v e$ symmetry but also satisfies desired condition, $\left.\Phi(r, R)^{+}\right|_{\mathrm{r}=0}=0$.

Before we proceed with the formulation of our theory further, it is important to speak about the analytic nature of $\psi_{\mathrm{q}}(\mathrm{r})^{-}$(Eqn.11) and $\varphi_{\mathrm{q}}(\mathrm{r})^{+}$[appearing, respectively, in Eqn.(10) and Eqn.(12)] which represent different possible states of the pair distinguished, viz., by different values of angular momentum, $l=0,1,2,3, .$. , identified, respectively, as s, $\mathrm{p}, \mathrm{d}, \ldots$ states. The s state of $\mathbf{q} \| \mathbf{r}$ with lowest $|\mathbf{q}|=q_{\mathrm{o}}$ represents the G-state of the pair because the pair has no motion other than zero point motion, while states with $l \neq 0$ have an additional motion (in addition to zero-point motion) represented by $\mathbf{q}_{\perp}$ (component of $\mathbf{q} \perp$ to $\mathbf{r}$ ) in addition to $\mathbf{q}_{\|}$ (component of $\mathbf{q} \|$ to $\mathbf{r}$ ) indicating that the net $\mathbf{q}=\mathbf{q}_{\|}+\mathbf{q}_{\perp}=$ $\mathbf{q}_{\mathrm{o}}+\mathbf{q}_{\perp}$. Evidently, even for a state of $l \neq 0$ we have $\mathbf{q} \cdot \mathbf{r}=\mathbf{q}_{\mathrm{o}} \cdot \mathbf{r}$ $+\mathbf{q}_{\perp} \cdot \mathbf{r}=\mathbf{q}_{\mathrm{o}} . \mathbf{r}$ which, does not vanish because $q_{\mathrm{o}}$ has non-zero value for every particle in our system (see Eqn.(16) as well as Section 3.3). This observation is discussed again in Section 6.3(i) in the light of an experimental support for it.

Analyzing $\Psi(r, R)^{-}$(Eqn.10) and $\Phi(r, R)^{+}$(Eqn.12), and related relations we note the following:

(i). The fact that Eqn. 6 renders

$$
\mathbf{p}_{1}=-\mathbf{q}+\mathbf{K} / 2 \text { and } \mathbf{p}_{2}=\mathbf{q}+\mathbf{K} / 2
$$

which, without any loss of generality, reveals that two bosons[fermions] in $\Phi(r, R)^{+}\left[\Psi(r, R)^{-}\right]$state can be identified as particles moving with equal and opposite momenta $(\mathbf{q},-\mathbf{q})$ with respect to their $\mathrm{CM}$ which moves freely as plane wave of momentum K. Since this agrees with the fact that $\psi_{\mathbf{q}}(\mathbf{r})^{-}$ (Eqn.11) and $\varphi_{\mathbf{q}}(\mathbf{r})^{+}$(Eqn.12) represent a standing wave[or what we call as standing matter wave (SMW)], we propose to call the pair in $\Psi(r, R)^{-}$and $\Phi(r, R)^{+}$states as $(\mathbf{q},-\mathbf{q})$ pair or SMW pair. In addition, we have $\left|\varphi_{\mathbf{q}}(\mathbf{r})^{+}\right|^{2}=\left|\psi_{\mathbf{q}}(\mathbf{r})^{-}\right|^{2}$ which reveals an important fact that bosonic/fermionic nature does 
not affect the relative configuration of two $\mathrm{HC}$ particles and implies that the laws of bosonic/fermionic distribution of particles should be implemented in relation to the energy levels of allowed $K$. We use this inference in constructing $N$ particle wave functions ( $c f$. Eqn.20).

(ii) A pair of particles in 3-D has six degrees of freedom. Three of them represent 3 independent components of a pure translatory motion of their $\mathrm{CM}$, while the rest of three motions include : (i) two components of a motion with $\mathbf{k} \perp$ to $\mathbf{r}$ and (ii) pure relative motion with $\mathbf{k} \|$ to $\mathbf{r}$; as discussed before, a combine of (i) and (ii) in general renders a planar motion, -a kind of rotation with $l=0$ (s-state) or $l \neq 0\left(\mathrm{p}^{-}, \mathrm{d}-, \mathrm{f}-\ldots \ldots\right.$ states depending on the value of $l$ ). Since $\mathbf{r}$ changes only in (ii), the interaction $V(r)$ affects only pure relative motion which evidently represents a 1-D motion, we can generalize our result $<\mathrm{A}(x) \delta(x)>=0$ (Appendix -A) to conclude that $<\Psi(r, R)^{-}|\mathrm{A}(r) \delta(r)| \Psi(r, R)^{-}>=<\Phi(r, R)^{+}|\mathrm{A}(r) \delta(r)| \Phi(r, R)^{+}>=0$

which renders

$$
E(2)=<H(2)>=\left(\hbar^{2} / 4 m\right)\left[k^{2}+K^{2}\right]=\left(\hbar^{2} / 2 m\right)\left[p_{1}{ }^{2}+p_{2}{ }^{2}\right]
$$

as the energy of two $\mathrm{HC}$ fermions/bosons. Note that $\langle H(2)>$ is independent of the use of $\Psi(r, R)^{-}$(Eqn.10) or $\Phi(r, R)^{+}$ (Eqn.12) as per the symmetry requirement of the states of two bosons/fermions. This agrees with the physical reality of a fluid that: (i) the energy of its particles is mainly kinetic and (ii) the interactions basically decide the inter-particle distance $d$ and the level of flat potential $-V_{\mathrm{o}}$ on which particles move freely. It may, however, be mentioned that this picture is largely valid at HT where quantum nature of particles has little or no impact on the behavior of the system. The picture changes at LT at which a combine of inter-particle interactions and wave superposition, plays a dominant role in modifying the behavior of the system by transforming a part of the kinetic energy of relative motions of particles into their zero-point energy[ $\varepsilon_{0}$ (Eqn.27) which represents a kind of potential energy for its dependence on $d$ and serves as the source of zero-point repulsion]. Thus it should be clear that our theory adequately incorporates interactions in reaching its conclusions and as shown in Section 4 and 5, one may find the method it uses interactions to reveal the origin of different properties of a SIB (including superfluidity and related aspects).

(iii) Analyzing the wave mechanics of two $\mathrm{HC}$ particles presumed to have 1-D motion we recently concluded[53] that the expectation value of their relative separation satisfies $<x>\geq \lambda / 2$. We use this inference with the fact that the pure relative motion of two particles (interacting through a central force) in 3-D space is equivalent to their pure relative motion in 1-D to conclude that $\mathrm{P} 1$ and $\mathrm{P} 2$ in their $\varphi_{\mathrm{k}}(\mathbf{r})^{+}$state (Eqn.12) should have $\langle r\rangle \geq \lambda / 2$ for $\mathbf{k} \| \mathbf{r}$ case and $\langle r\rangle \geq \lambda / 2 \cos \theta$ in general; here $\theta$ is the angle between $\mathbf{k}$ and $\mathbf{r}$ ). This means (as $|\cos \theta| \leq 1)$ that two $\mathrm{HC}$ particles from an experimental view point can reach a shortest distance of $\langle r\rangle_{0}=\lambda / 2$ only and in this situation their individual locations from their $\mathrm{CM}$ are given by $\left\langle r_{\mathrm{CM}}(1)\right\rangle_{\mathrm{o}}=-\left\langle r_{\mathrm{CM}}(2)\right\rangle_{\mathrm{o}}=\lambda / 4$. Using similar result for their shortest possible distance on the $\varphi$-line $(\varphi=\mathbf{k} . \mathbf{r})$, we note that $\Phi(r, R)^{+}$state is characterized by

$$
\lambda / 2 \leq<r>=d \text { or } q>q_{\mathrm{o}}(=\pi / d)
$$

with $d$ being the nearest neighbor distance of two particles in a SIB and

$$
<\Delta \varphi>\geq 2 \pi
$$

\subsection{Macro-orbital Representation of A Particle}

As evident from Eqn.15, the wave superposition of P1 and P2 leading to $\Psi(r, R)^{-}$(Eqn.10) or $\Phi(r, R)^{+}$(Eqn.12) does not alter their net energy $E(2)=\hbar^{2} p_{1}{ }^{2} / 2 m+\hbar^{2} p_{2}{ }^{2} / 2 m$ in their states represented by two plane waves. This implies that: (i) $\mathrm{P} 1$ and $\mathrm{P} 2$ in $\Psi(r, R)^{-} / \Phi(r, R)^{+}$states behave, energetically, like independent particles, (ii) $E(2)$ is shared equally among $\mathrm{P} 1$ and P2 and (iii) the superposition brings P1 and P2 (having different energies, $E_{1}=\hbar^{2} p_{1}{ }^{2} / 2 m$ and $E_{2}=\hbar^{2} p_{2}{ }^{2} / 2 m$ ) to a single energy state of $\left(E_{1}+E_{2}\right) / 2$ (cf., Fig.1). Evidently, each of the two fermions/bosons, in $\Psi(r, R)^{-} / \Phi(r, R)^{+}$state, can be identified as an independent particle in a state represented by

$$
\xi(i)=\zeta\left(r_{i}\right) \exp \left(i \mathbf{K}_{i} \cdot \mathbf{R}_{i}\right) \text { with } \zeta\left(r_{i}\right)=\sin \left(\mathbf{q}_{i} \cdot \mathbf{r}_{i}\right)
$$

where subscript $i$ refers to the $i$-th particle $(i=1$ or 2$)$ of a pair it represents. Since $-v e(+v e)$ sign of $\psi_{\mathbf{k}}(\mathbf{r})^{-}\left(\varphi_{\mathbf{k}}(\mathbf{r})^{+}\right)$ (Eqn.10/12) loses significance in the light of $\left.\psi_{\mathbf{k}}(\mathbf{r})^{-}\right|^{2}=$ $\left|\varphi_{\mathbf{k}}(\mathbf{r})^{+}\right|^{2}, \xi(i)$ can be used identically to represent a fermion or a boson. We call $\xi(i)$ a macro-orbital because it serves as a basis for the macroscopic 3-D network of SMWs ( $c f$. Section 3.3 , below) which enfolds the entire system in its S-state and there by helps in understanding the manifestations of wave nature of particles at macroscopic level. A brief discussion (Section 6.3) reveals how experimental reality of the existence of an electron bubble renders strong support for the accuracy of $\xi(i)$ in describing the low energy states of a ${ }^{4} \mathrm{He}-$ atom in LHe-4 having zero and non-zero angular momenta.

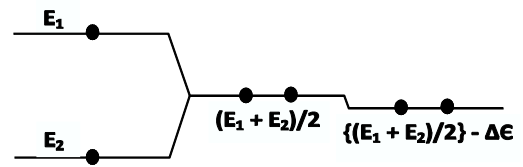

Figure 1. Three physical stages of two quantum particles: (Left) in plane wave states of momenta $k_{1}$ and $k_{2}$ (or energy $E_{1}$ and $E_{2}$, respectively), (Middle) in pair state $\Psi(r, R)^{-}$(Eqn.10) or $\Phi(r, R)^{+}$(Eqn.12) where each of them has $\left(E_{1}+E_{2}\right) / 2$ energy, and (right) in bound pair state with binding energy $\Delta \epsilon=2|\beta \mathrm{o}(\mathrm{T})|($ See Section 5.1)

The fact that a particle (say $i-$ th) in a many body quantum systems (MBQS) naturally assumes a physical state represented by a macro-orbital (Eqn.18) can also be established by tracking its motion when it collides with another particle (or a set of particles or a boundary of the fluid); note that the particle, before and after such a collision, can have different momenta, say, $\mathrm{p}_{i}$ and $\mathrm{p}_{i}^{\prime}$ indicating that its state $\left(\psi(\mathrm{i})^{ \pm}\right)$ around the point of collision should be represented by the superposition of $\mathrm{u}_{\mathrm{p} i}\left(\mathrm{~b}_{i}\right)$ and $\mathrm{u}_{\mathrm{p}^{\prime} \mathrm{i}}\left(\mathrm{b}_{i}^{\prime}\right)$ which can be expressed by

$$
\psi(i)^{ \pm}=\mathrm{u}_{\mathbf{p} i}\left(\mathbf{b}_{i}\right) \mathrm{u}_{\mathbf{p}^{\prime} i}\left(\mathbf{b}_{i}^{\prime}\right) \pm \mathrm{u}_{\mathbf{p} i}\left(\mathbf{b}_{i}^{\prime}\right) \mathrm{u}_{\mathbf{p}^{\prime} i}\left(\mathbf{b}_{i}\right)
$$

Since $\psi(i)^{-}$, vanishing at $r=0$ (the point of collision), it rightly represents the state of the chosen particle (independent of its fermionic/bosonic nature) which, obviously, 
proves that $\psi(i)^{-} \equiv \xi(i)$.

Here we note that the macro-orbital state (Eqn.18) of a particle has following important aspects.

(1). MS and SS states : In what follows from this discussion, Eqn.18 virtually represents the superposition of two plane waves which may either be identified with two different particles, P1 and P2, or with two states of the same particle. Obviously, in the former case we have a state of mutual superposition (MS) of two particles, while in the latter case we have the self superposition (SS) of the same particle. However, we have no means to fix whether P1 and P2 have their MS or each one of them (after the collision) falls back on itself and assumes its SS state. Guided by this fact, we can always identify each of the two particles (in a state of their wave superposition) in its SS state and represent it by a macro-orbital. As demonstrated in this paper, the macro-orbital representation of a particle emerges as a simple tool which greatly simplifies the mathematical formulation of the microscopic theory of a MBQS. A more detailed discussion on MS and SS states can be seen in our recent paper[53].

(2). Two motions : Each particle in a macro-orbital state has two motions ( $q$ and $K$ ) which are, respectively, related to the relative and $\mathrm{CM}$ motions of the pair, -it represents. Since only relative motion encounters a potential such as $\mathrm{A}(r) \delta(r)$, the possible values of only $q$ are restricted by the interaction (cf., Appendix - A). The $K$-motion is not expected to have any impact of the interaction and $K$ can have any value between 0 and $\infty$.

\section{N-particle State}

\subsection{State Function}

Using $N$ macro-orbitals for $N$ particles, we obtain $\Psi_{\mathrm{n}}^{j}(N)=\prod_{i}^{N \zeta}\left(r_{i}\right)\left[\Sigma_{P}^{N !}( \pm 1)^{P} \prod_{i}^{N} \exp \left(i P \mathbf{K}_{i} \cdot \mathbf{R} i\right)\right]$

as the state function, presumably, representing $j$-th micro-state of the system. Here $\Sigma_{P} N$ ! refers to the sum of $N$ ! product terms obtained by permuting $(P) N$ particles on different $\mathbf{K}_{i}$ states. We use $(+1)^{P}$ (or $(-1)^{P}$ ) depending on the bosonic/fermionic nature of the system to ensure symmetric/anti-symmetric nature of $\Psi_{\mathrm{n}}^{j}(N)$ for an exchange of two particles. Since the permutation of $N$ particles on different $q_{i}$ states also gives $N$ ! different $\Psi_{n}^{j}(N)$, we have

$$
\Phi_{n}(N)=(1 / N !) \Sigma_{j}^{N !} \Psi_{\mathrm{n}}^{j}(N)
$$

as the complete wave function of a possible quantum state of a bosonic/fermionic system.

\subsection{State Energy}

We define

$$
h(i)=\left(h_{i}+h_{\mathrm{i}+1}\right) / 2=-\left(\hbar^{2} / 4 m\right)\left[\nabla_{R i}^{2}+4 \nabla_{r i}^{2}\right]
$$

with $h_{N+1}=h_{1}$ and write Eqn.(2) as

$$
H_{\mathrm{o}}(N)=\Sigma_{i}^{N} h(i)+\Sigma_{i>j}{ }^{N} \mathrm{~A}\left(r_{i i}\right) \delta\left(r_{i j}\right)
$$

Using Eqns. 21 and 14, we easily obtain

$$
<\Phi_{n}(N)\left|\Sigma_{\mathrm{i}>\mathrm{j}} \mathrm{j}^{N} \mathrm{~A}\left(r_{i i}\right) \delta\left(r_{i j}\right)\right| \Phi_{n}(N)>=0
$$

and

$$
E_{n}(N)=<\Phi_{n}(N)\left|H_{\mathrm{o}}(N)\right| \Phi_{n}(N)>=\left(\hbar^{2} / 8 m\right) \Sigma_{i}^{N}\left[K_{i}^{2}+4 q_{i}^{2}\right]
$$

which concludes that $\Phi_{n}(N)$ is an eigen function of $H_{0}(N)$ with $E_{n}(N)$ eigen energy.

\subsection{Ground State}

With a view to determine the G-state energy of our system, we first use our inference that $q \geq q_{\text {o }}$ (Eqn.16) and the fact that $K$ can have any value between 0 and $\infty$ to find

$\mathrm{E}_{q i, K i=0}(N)=\Sigma_{i}^{N} h^{2} / 8 m d_{i}^{2}=\Sigma_{i}^{N} h^{2} / 8 m v i^{2 / 3}$ with $\Sigma_{i}^{N} v_{i}=\mathrm{V}$ (26)

by putting $q_{i}=\pi / d_{i}\left(\approx q_{\mathrm{o}}\right)$ and $K_{i}=0$ for all particles in Eqn. 25 with $d_{i}=v_{i}{ }^{1 / 3}$ where $v_{i}$ represents the volume of the real space, exclusively occupied by $i$-th particle. In writing $\Sigma_{\mathrm{i}}^{N} v_{i}=\mathrm{V}$, we use the fact that each particle of the lowest possible $q=q_{i}$ has largest possible quantum size $\lambda_{i} / 2$ for which it occupies largest possible $v_{i}$ exclusively. While, different particles can, in principle, occupy different volumes but simple algebra reveals that $E_{q i, K=0}(N)$ assumes its minimum value for $v_{1}=v_{2}=. . v_{\mathrm{N}}=\mathrm{V} / \mathrm{N}$ and this renders

$$
E_{\mathrm{o}}(N)=N h^{2} / 8 m d^{2}=N \varepsilon_{\mathrm{o}}
$$

as the desired G-state energy where $\varepsilon_{\mathrm{o}}=h^{2} / 8 m d^{2}$ represents G-state energy of a single particle. Since all particles in the G-state, identically, have $q=q_{\mathrm{o}}$ and $K=0$ which can be used in Eqn.20/21 to obtain

$$
\Phi_{\mathrm{o}}(N)=\Pi_{i}^{N \zeta_{q o}}\left(r_{i}\right)=\Pi_{i}^{N} \sin \left(q_{\mathrm{o}} r_{i}\right)
$$

as the G-state wave function. It is clear from Eqns.27 and 28 that each particle in the G-state represents a particle trapped in a box (cavity formed by its neighboring particle) of size $\mathrm{d}$ and it rests at the central point of this cavity. Since, each $\sin \left(q_{\mathrm{o}} r_{\mathrm{i}}\right)$ in Eqn.28 represents a kind of SMW which joins with other SMWs of neighboring particles at the boundaries between the two cavities, the G-state wave function seems to be a macroscopically large size 3-D network of SMWs which modulates the relative positions of two particles in the phase space and extends from one end of the container to another. We find that this network gets energetically stabilized due to some kind of collective binding (Section 5.0) and assumes different aspects of macroscopic wave function of the S-state, as envisaged by London[11]. In view of this inference, Eqns. 16 and 17 imply that the G-state configuration of particles in a SIB satisfies

$$
<k>=0, \quad<r>=d \quad \text { and }\langle\Delta \varphi>=2 n \pi
$$

with $n=1,2,3$. Although, each particle in the G-state of a SIB retains a zero-point energy $\varepsilon_{0}$ (from which one can derive zero-point momentum $q_{\mathrm{o}}$ ), but as evident from $<k>=0$, particles in the G-state cease to have relative motions which represent the main cause of inter-particle collisions, scattering and non-zero $\eta$ ). This explains why G-state should be a state of $\eta=0$. However, this situation does not forbid particles from moving in coherence with equal velocity without any change in order of their locations. Since all particles assume $\langle r\rangle=d$ (nearest neighbor distance), the G-state represents a close packed arrangement of their wave packets (CPA-WP) in real space which can, obviously, sustain phonon-like collective motions as the quasi-particle excitations of the system. 


\section{4. $\lambda$-Transition and Related Aspects}

\subsection{Equation of State}

In what follows from Section 3 and Eqn.(15), energy $(\epsilon)$ of a single particle can be written as

$$
\epsilon=E(2) / 2=\epsilon(K)+\epsilon(k)=\left(\hbar^{2} / 8 m\right)\left[K^{2}+k^{2}\right]
$$

which can have any value between $\varepsilon_{0}$ and $\infty$. Since, this possibility exists even if $h^{2} k^{2} / 8 m$ is replaced by $\varepsilon_{0}$ (the lowest energy of $q$-motion) because $K$ can have any value between 0 and $\infty$, we can use

$$
\epsilon=\left(\hbar^{2} K^{2} / 8 m\right)+\varepsilon_{0}
$$

in the starting expressions of the standard theory of BEC[9, Ch.7] to obtain the equation of state of our system. We have

$$
\begin{aligned}
& P V / k_{B} T=-\Sigma_{\epsilon(K)} \ln \left[1-z \exp \left(-\beta\left\{\epsilon(K)+\varepsilon_{0}\right\}\right)\right] \\
& \left.N=\Sigma_{\epsilon(K)} z^{-1} \exp \left(-\beta\left\{\epsilon(K)+\varepsilon_{0}\right\}\right)-1\right]^{-1} \\
& \text { with } \beta=1 / k_{B} T \text { and fugacity } \\
& z=\exp (\beta \mu) \\
& \text { with } \mu=\text { chemical potential }
\end{aligned}
$$

Once again, by following the steps of the standard theory of BEC[9] and redefining the fugacity by

$$
z^{\prime}=z \exp \left(-\beta \varepsilon_{0}\right)=\exp \left[\beta\left(\mu-\varepsilon_{0}\right)\right]=\exp \left(\beta \mu^{\prime}\right)
$$

with $\mu^{\prime}=\mu-\varepsilon_{0}$. We easily have

$$
P / k_{B} T=\left(\lambda_{T}^{\prime}\right)^{-3} g_{5 / 2}\left(z^{\prime}\right)
$$

and

$$
\left(N-N_{\mathrm{o}}\right) / \mathrm{V}=\left(\lambda_{T}^{\prime}\right)^{-3} g_{3 / 2}\left(z^{\prime}\right)
$$

where we have $\lambda_{\mathrm{T}}^{\prime}=h /\left(2 \pi(4 m) k_{B} T\right)^{1 / 2}$ and $\mathrm{g}_{\mathrm{n}}\left(z^{\prime}\right)$ having its usual expression[9]. This reduces a SIB to a SNIB (system of non-interacting bosons), but with a difference. Firstly, $m$ is replaced by $4 m$ and $z$ by $z^{\prime}$. Secondly, the theory of a SNIB concludes $z=1$ ( or $\mu=0$ ) for $T \leq T_{\lambda}$ and $z<1$ (or $\mu<0$ ) for $T>$ $T_{\lambda}$ while our theory fixes $z^{\prime}=1$ [or $\mu^{\prime}=0$ or $\mu=\varepsilon_{0}$ (Eqn.35)] for $T \leq T_{\lambda}$ and $\mathrm{z}^{\prime}<1$ [or $\mu^{\prime}<0$ or $\mu<\varepsilon_{0}$ ] for $T>T_{\lambda}$. In other words, we have $z^{\prime}$ and $\mu^{\prime}$ in place of $z$ or $\mu$ used in the theory of a SNIB[9]. As such, we can use Eqns.32 and 33 or Eqns. 36 and 37 to evaluate different thermodynamic properties of a SIB. For example, using Eqns.36 and 37, we find its internal energy by using, $U=-\left.\partial_{\beta}\left(P \mathrm{~V} / k_{B} T\right)\right|_{z, \mathrm{~V}}$. We have

$$
U=(3 / 2) k_{B} T \mathrm{~V} \lambda_{T}^{\prime-3} g_{5 / 2}\left(z^{\prime}\right)+N \varepsilon_{\mathrm{o}} .=U^{\prime}+N \varepsilon_{\mathrm{o}}
$$

where $U^{\prime}=-\left.\partial_{\beta}\left(P V / k_{B} T\right)\right|_{z^{\prime}, \mathrm{V}}$, obviously, represents the internal energy of $K$-motions, while $N \varepsilon_{0}$ comes from $q$-motions. Similarly, by using $\mu=\mu^{\prime}+\varepsilon_{0}$, we find that the Helmholtz free energy of a SIB can be obtained from

$$
F=N \mu-P \mathrm{~V}=N \varepsilon_{0}+\left(N \mu^{\prime}-P \mathrm{~V}\right)=F\left(q=q_{\mathrm{o}}\right)+F(K)
$$

Although, to find more accurate relations for $U$ and $F$ one needs to consider the facts that small fraction of particles in the system can have $q=2 q_{\mathrm{o}}, 3 q_{\mathrm{o}} \ldots$, etc. (a rough estimate reveals that this fraction falls $\approx 5 \%$ at $T_{\lambda}$ ) and the real dispersion of $K$-motion may differ from $\epsilon(K)=\hbar^{2} K^{2} / 2(4 m)$ due to inter-particle correlations which are expected to render phonon like dispersion; however, we plan to consider these aspects in our forthcoming paper.

\subsection{Onset of $K=0$ Condensate and $T_{\lambda}$}

In what follows from Eqns.36 and 37, we can apply the standard theory of a SNIB[9] to conclude that a SIB should exhibit a transition at

$$
T_{\mathrm{b}}=T_{\mathrm{BEC}} / 4=\left(h^{2} / 8 \pi m k_{B}\right)(N / 2.61 \mathrm{~V})^{2 / 3}
$$

which could be identified with the onset of $K=0$ condensate in a gas of non-interacting quasi-particles. Here $T_{\mathrm{BEC}}$ represents the usual temperature of BEC[9] in a SNIB and $1 / 4$ factor signifies that each boson for its $K$-motion behaves like a particles of mass $4 m$. However, as the derivation of Eqn.40, excludes $q$-motions of particles, to get $T_{\lambda}$ (the real transition temperature), $T_{\mathrm{b}}$ should be added to

$$
T_{\mathrm{o}}=h^{2} / 8 \pi m k_{B} d^{2}
$$

which represents the $T$ equivalent of $\varepsilon_{0}$ because $N \varepsilon_{0}$ component of $U$ (Eqn.38) and $F$ (Eqn.39) too comes from the thermal energy of the system. In finding Eqn.(41), we use $\lambda_{T}$ $=2 d$ (with $\lambda_{T}=h / \sqrt{ }\left(2 \pi m k_{B} T\right)$ being the thermal de Broglie wave length[5]), since $\varepsilon_{0}$ for each particle corresponds to $\lambda=$ $2 d$. This renders

$$
T_{\lambda}=T_{\mathrm{o}}+T_{b} \approx 1.53 T_{\mathrm{o}}
$$

which clearly differs from that of a SNIB. While, $K=0$ condensation of particles in a SIB occurs in its G-state characterized by non-zero energy $\left(\varepsilon_{0}\right)$, the $p=0$ condensation in a SNIB occurs in the G-state of zero energy. In addition while particles in a SIB are representatives of $(\mathbf{q},-\mathbf{q})$ pairs, those in a SNIB are simply independent particles which have no mechanism to identify the presence of each other.

\subsection{Nature of Transition}

Following Section 3.3, we find that the system transforms from a state of random distribution of its particles in $\varphi$-space to that of orderly distribution with $\varphi=2 n \pi(n=1,2,3, .$.$) ,$ when it is cooled through $T_{\lambda}$. Evidently, the $\lambda$-transition is an onset of order-disorder of particles in $\varphi$-space followed by the BEC of particles in the state of $K=0$ and $q=\pi / d$. We note that the BEC of particles in $K=0$ state is not different from the BEC of non-interacting bosons (a well known second order transition[9]) and the order-disorder of particles in $\varphi$-space is accomplished simply by a reshuffle of their momenta (kinetic energy) without any change in inter-particle distance and potential energy; this means that there is no change in the total energy of the system at $\lambda$-point which concludes it to be second order transition.

\subsection{Free Energy and Order Parameter of $T_{\lambda}$}

Following Section 4.1, we have

$$
F=F(q)+F(K) \approx N \varepsilon_{0}+F(K)
$$

with

$$
F(K)=\left(k_{B} T / \lambda_{\mathrm{T}}^{\prime}\right) g_{5 / 2}\left(z^{\prime}\right)
$$

To a good approximation Eqn.43 is valid even for $T=T_{\lambda}^{+}$ (just above $T_{\lambda}$ ) since almost $95 \%$ particles in the system at $T_{\lambda}$ are estimated to have $\varepsilon_{0}$ energy. Guided by this fact and the expected occurrence of $\lambda$-transition (cf. Section 4.2), $F$ can be assumed to be a function of $\Omega$, -an order parameter (OP) of the transition and be expanded as

$$
F(T, \Omega)=F_{\mathrm{o}}+(1 / 2) A \Omega^{2}+(1 / 4) B \Omega^{4}+(1 / 6) C \Omega^{6}
$$

where $F_{\mathrm{o}}$ is independent of $T$ and $P$, while the coefficients, $A, B, C$, etc., may, in principle, depend on $T$ and $P$. Since, $F(K)$ (Eq.44) representing a kind of SNIB vanishes at $T=0$ where $\rho_{\mathrm{s}} / \rho=1.0$ (i.e. $100 \%$ S-component). Evidently, superfluidity and related properties of a SIB have their origin in $F(q)$ only; this, obviously, means that a SNIB is not expected 
to exhibit superfluidity below $T_{b}$ (Eqn.40), although, it is concluded to have BEC[9]. Assuming $A=\alpha\left(T-T_{\lambda}\right)$ and $B$ being independent of $T$ and $+v e$ with other terms having powers of $\Omega$ higher $>4$, we find

$$
\Omega_{\mathrm{R}}(T)=(T) / \Omega(0)=\sqrt{ }\left[\left(T_{\lambda}-T\right) / T_{\lambda}\right]
$$

which represents $\Omega(T)$ (in units of $\Omega(0)$ ) assumed by the system in its LT phase. Since, almost all particles at $T=T_{\mathrm{o}}$ occupy their G-state with $\varepsilon_{0}$ energy, the system hardly retain any energy with its excitations. This implies that a SIB at $T=$ $T_{\mathrm{o}}$ is effectively in a $T=0$ state for which $\Omega\left(T_{\mathrm{o}}\right) \approx \Omega(0)$. Guided by this inference we re-normalize the $T$-scale by replacing $T$ in Eqn. 46 by $T^{*}=T-T_{\mathrm{o}}$ and recast $\Omega_{\mathrm{R}}(T)$ as

$$
\Omega_{\mathrm{R}}\left(T^{*}\right)=\sqrt{ }\left[\left(T_{\lambda}^{*}-T^{*}\right) / T_{\lambda}^{*}\right]=\sqrt{ }\left[\left(T_{\lambda}-T\right) /\left(T_{\lambda}-T_{\mathrm{o}}\right)\right]
$$

which rightly reaches its maximum value 1.0 at $T=T_{\mathrm{o}}$.

As observed for LHe-4, a number of physical properties (viz., a-c, as listed below) of a SIB for their relation with (T) can be expected to exhibit notable dependence on $T$ around $T_{\lambda}$.

(a) The fraction of the number of bosons, $n_{K=0}(T)=$ $N_{K=o}(T) / N$, condensed into $q=q_{\mathrm{o}}$ and $K=0$ state.

(b) The fractional number of particles, $n^{*}\left(T \leq T_{\lambda}\right)$ $\left.=\left[N^{*}\left(T_{\lambda}\right)-N^{*}(T)\right] / N^{*}\left(T_{\lambda}\right)\right]$ which increases smoothly from $n^{*}\left(T_{\lambda}\right)=0$ to $n^{*}(0)=1$ in a manner the OP of the transition is expected to increase with fall in $T$ below $T_{\lambda}$. Here we define $\left.N^{*}(T)=\mathrm{A} \int_{\varepsilon c} \exp \left\{(\varepsilon-\varepsilon 0) / k_{B} T\right\}-1\right]^{-1} \sqrt{\varepsilon} \mathrm{d} \varepsilon$

[with $\mathrm{A}=\left(\mathrm{V} / 4 \pi^{2}\right)\left(2 m / \mathrm{h}^{2}\right)^{3 / 2}$ ] as the number of particles in excited states of energy, $\varepsilon \geq \varepsilon_{\mathrm{c}}\left(=\hbar^{2} Q_{\mathrm{c}}^{2} / 2 m\right.$, with $\left.Q_{\mathrm{c}}=2 \pi / \sigma\right)$; to a good approximation these states correspond to single particle motion, since the excitation wave length $\Lambda \leq \sigma(<d)$ which implies that the impact of the excitation is localized to a space shorter than the inter-particle distance indicating that the energy and momentum of the excitation is carried basically by a single particle. These states, obviously, lack in inter-particle $r^{-}, k-$ and $\varphi$-correlations [54] which are found to be basic character of particles in the G-state and $n^{*}(T)$ $\left.=\left[N^{*}\left(T_{\lambda}\right)-N^{*}(T)\right] / N^{*}\left(T_{\lambda}\right)\right]$ is a measure of the number of particles which fall from a state having no inter-particle correlations to the G-state having well defined correlations (Eqn.29).

(c) The volume expansion of the system forced by zero-point force on cooling through $T_{\lambda}$ which can be viewed as the origin of strain in inter-particle bonds, defined by $\delta(T)$ $=\Delta d / d_{\lambda}=\left(d_{T}-d_{\lambda}\right) / d_{\lambda}$, etc. where $d_{T}$ and $d_{\lambda}$, respectively, represent $d$ at a chosen $T<T_{\lambda}$ and $T_{\lambda}$.

Since particles in a SIB for their $K$-motions behave like non-interacting bosons, we evaluate $n_{K=0}(T)$ (identified in (a) above) by using $n_{K=0}(T)=\left[1-\left(T / T_{\lambda}\right)^{3 / 2}\right]$ obtained from the standard theory of BEC of a SNIB[9]. Recasting $n_{K=0}(T)$ on the $T^{*}$-scale, we obtain its equivalent for a SIB given by

$$
\begin{gathered}
n_{K=0}\left(T^{*}\right)=N_{K=0}\left(T^{*}\right) / N=\left[1-\left(T^{*} / T^{*} \lambda\right)^{3 / 2}\right]=\left[1-\left\{\left(T-T_{\mathrm{o}}\right) /\left(T_{\lambda}\right.\right.\right. \\
\left.\left.\left.-T_{\mathrm{o}}\right)\right\}^{3 / 2}\right]
\end{gathered}
$$

Defining $t=T / T_{\lambda}$, we depict in Fig.2: (i) $n_{K=0}(t)=\left(1-t^{3 / 2}\right)$ which can be applied to $K$-motions of particles in a SIB as for these motions they behave like particles in a SNIB, (ii) $\left.n^{*}(t)=\left[N^{*}\left(T_{\lambda}\right)-N^{*}(T)\right] / N^{*}\left(T_{\lambda}\right)\right]$ (obtained by using Eqn.48) and (iii) $\Omega_{\mathrm{R}}(T)$ (Eqn.46), respectively, by Curves A, B and C. We also plot $n_{K=0}\left(t^{*}\right)$ (Eqn.49) and $\Omega_{\mathrm{R}}\left(T^{*}\right)$ (Eqn.47), respec- tively, by Curves $\mathrm{A}^{*}$ and $\mathrm{C}^{*}$ as well as $\delta(\mathrm{t})$ (Curve E1)[defined in point (c) above] and $\rho_{\mathrm{s}}(t) / \rho$ (Curve E2) for LHe-4. Since, $\delta(t)$ and $\rho_{\mathrm{s}}(t) / \rho$ are obtained, respectively, by using experimental values of $T$-dependence of molar volume[2(a)] and second sound velocity[5] of LHe-4, they represent a measure of experimental OP of LHe-4. While, the agreement of Curves E1 and E2 closely with Curve $\mathrm{C}^{*}$, Curve $\mathrm{B}$ and Curve $\mathrm{A}^{*}[c f$., Fig.2] establishes the importance of $T_{\mathrm{o}}$ for the effective $T$-dependence of the OP of the $\lambda$-transition in LHe-4, their disagreement with Curves A and $\mathrm{C}$ concludes that interactions really make the nature of BEC in a SIB to differ from the nature of BEC in a SNIB[9]. Evidently, this comparison provides strong experimental proof that particles in a SIB fall into their $q=q_{\mathrm{o}}$ state of energy $\varepsilon_{0} \equiv k_{B} T_{\mathrm{o}}$ on its cooling through $T_{\lambda}$.

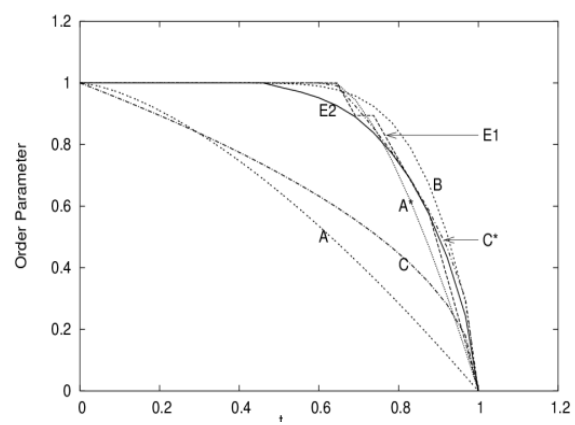

Figure 2. $t=T / T_{\lambda}$ dependence of different representatives of the order parameter of $\lambda$-transition: $n_{K=0}(t)=1-t^{3 / 2} \quad$ (Curve-A), $n^{*}(t)$ $\left.=\left[N^{*}\left(T_{\lambda}\right)-N^{*}(T)\right] / N^{*}\left(T_{\lambda}\right)\right]$ (Curve-B, obtained by using Eqn.48), $\Omega_{\mathrm{R}}(t)$ (Curve-C, obtained from Eqn.46), $n_{\mathrm{K}=0}\left(t^{*}\right)\left(\right.$ Curve-A*, Eqn.49), $\Omega_{\mathrm{R}}\left(t^{*}\right)$ (Curve-C ${ }^{*}$, Eqn. 47), $\delta(t)$ [defined in point-(c) of Section 4.4 and plotted as Curve-E1, obtained from experimental volume expansion data[2(a)]), and $\rho_{\mathrm{s}}(t) / \rho$ (Curve-E2, obtained from experimental superfluid density of LHe-4[5]). For further identification see last paragraph of Section 4.4

Interestingly, since the state of a particle with $q=q_{\mathrm{o}}$ (represented by $\left.\psi_{\mathrm{q}}(r)=\sin \left(q_{\mathrm{o}} r\right)\right)$ is an eigen state of its kinetic energy operator $\left(\left(-\hbar^{2} / 2 m\right) \partial_{r}{ }^{2}\right)$ rather than its momentum operator, $-i \hbar \partial_{r}$, our reference to the momentum of the particle, basically, uses the fact that its value can be determined from its relation with kinetic energy of the particle; else, the momentum is not a good quantum number of its state. Further, because the expectation value of the momentum operator of the particle in this state, $\left\langle\psi q(r)\left|i \hbar \partial_{r}\right| \psi q(r)>\right.$, has zero value, Curve-A* (representing the growth in the number of particles condensed into the state of $q=q_{\mathrm{o}}$ and $K=0$ with decreasing T) can also be seen as the growth of number of bosons in the state of $\langle p\rangle=\hbar\langle q\rangle=0$. In this context we also note that the G-state of a particle in a SNIB confined to a finite $\mathrm{V}$ also corresponds to a state of $\langle p\rangle=\hbar\langle q\rangle=0$ and non-zero $p=p_{\mathrm{o}}=\hbar q_{\mathrm{o}} \approx h / 2 \mathrm{~V}^{2 / 3}$. When this physical reality is accepted (even for macroscopically large V), the BEC of bosons in a SNIB as well as in a SIB can be described identically (i.e., as a condensation of bosons in a state of $\langle p\rangle=0$, not of $p=0$ ). Since $T_{\mathrm{o}}$ for a SNIB has vanishingly small value, $\mathrm{BEC}$ of such bosons should, obviously, follow Curve A in place of Curve $A^{*}$ for a SIB.

\subsection{Single Particle Density Matrix and ODLRO}


The single particle density matrix $\rho_{1}\left(R^{\prime}-R\right)$, -a measure of the probability of spontaneous motion of a particle from $R$ to $R^{\prime}$ or vice versa assumes significance at $T \leq T_{\lambda} . \rho_{1}\left(R^{\prime}-R\right)$ is also known as the measure of off diagonal long range order (ODLRO). To obtain $\rho_{1}\left(R^{\prime}-R\right)$ for our system, we note that: (i) to a good approximation, almost all particles in a SIB[in its LT phase as well as at $T_{\lambda}^{+}$(just above $T_{\lambda}$ ) in HT phase] have $(\mathbf{q},-\mathbf{q})$ pair state with $q=q_{\mathrm{o}}$ and (ii) such a particle, when made to move with momentum $\Delta \mathbf{q}$, assumes a state of $(\mathbf{q}+\Delta \mathbf{q},-\mathbf{q}+\Delta \mathbf{q})$ which not only implies that its state changes from

$$
\sin (\mathbf{q} \cdot \mathbf{r}) \rightarrow \sin (\mathbf{q} . \mathbf{r}) \exp (\mathbf{i K} \cdot \mathbf{R}) \text { with } \mathbf{K}=2 \Delta \mathbf{q}
$$

but also concludes that particles with $K=0$ [i.e., whose states are defined simply by $\sin (\mathbf{q} . \mathbf{r})]$, are basically localized particles (each one having its wave packet (WP) spread over a space of size $\lambda / 2=\pi / q_{\mathrm{o}}=d$; the number of such particles $\left(N_{K=0}\left(T^{*}\right)\right)$, grows from its zero value (in HT phase) to a macroscopically large fraction in LT phase. Guided by these observations we easily obtain

$$
\rho_{1}\left(\mathbf{R}^{\prime}-\mathbf{R}\right)=\left[N_{K=0}\left(T^{*}\right) / \mathrm{V}+(N / \mathrm{V}) \exp \left[-\pi\left|\mathbf{R}^{\prime}-\mathbf{R}\right|^{2} / \lambda_{\mathrm{T}}^{\prime}\right] \sin ^{2}\left(q_{\mathrm{o}} r\right)\right.
$$

by separating particles having $K=0$ from those with $K \neq 0$ and evaluating the second term on the right by following the standard procedure for non-interacting bosons [9] which is justified because $K$-motions encounter no interactions. The $\left(\sin ^{2} q_{\mathrm{o}} r\right)$ term in Eqn.51 represents how probability density varies around the center of a particle WP in the cavity (size $d$ ) formed by neighboring particles. In what follows from Section 4.4, we use $N_{K=0}\left(T^{*}\right)$ (Eqn.49) in Eqn.51 rather than $N_{K=0}$ $(T)=N\left[1-\left(T / T_{\lambda}\right)^{3 / 2}\right]$ found for a SNIB[9].

Based on the value of $N_{K=0}\left(T^{*}\right)$, we find that $\rho_{1}\left(R^{*}-R\right)$ (under the limit $\left|\mathbf{R}^{*}-\mathbf{R}\right| \rightarrow \infty$ ) has nonzero and zero values, respectively, for LT and HT phases of a SIB. This proves that our theory is consistent with: (i) the criterion of Penrose and Onsager[55] for the occurrence of BEC which is shown to occur in $K=0$ and $q=\pi / d$ state which represents the G-state of a SIB and (ii) the idea of ODLRO advanced by Yang[56]. Further the fact that the particles at $T=T_{\lambda}$ move from a state of disorder $\Delta \varphi \geq 2 n \pi$ in $\varphi$-space to that of order $\Delta \varphi=2 n \pi$ concludes that our theory also agrees with spontaneous symmetry breaking and phase coherence as envisaged, respectively, by Goldstone[57] and Anderson[58].

\subsection{Evolution of the System on Cooling}

The q-motion state of a particle in the system can be characterized by a parameter $\mathrm{s}=d-\lambda / 2$ which decreases with cooling of the system since $\lambda_{\mathrm{T}} / 2=h / 2 \sqrt{ }\left(2 \pi m k_{B} T\right)$ (thermal average of $\lambda / 2$ ) increases and $d$ remains constant (or nearly constant). Evidently, since $\mathrm{s}$ is restricted to have only $+v e$ value in the light of the condition $\lambda / 2 \leq d$ (Eqn.16), the number of particles occupying a state of $\mathrm{s}=0$ (i.e., $\lambda=2 d$ or $q=q_{0}=\pi / d$ ) keep on increasing with falling $T$ till the system reaches a point where it satisfies $\lambda_{T} / 2=d$ (a condition which means that all particles, on an average, occupy the state of $\mathrm{s}=$ 0 ). As soon as, this happens, particles cease to have a possibility of any increase in $\lambda / 2$ because it demands increase in $d$ which is decided independently by $V\left(r_{i j}\right)$. Evidently, $\lambda / 2$ of particles at this point gets locked at $d$ or equivalent $q$ at $q_{\mathrm{o}}=$ $\pi / d$ indicating that $q-$ motions of particles are left with no energy to lose. Naturally, this forces particles to lose their $K$-motion energy for which the system sees an onset of their condensation in a state of $q=q_{\mathrm{o}}$ and $K=0$ at $T_{\lambda}$ given by Eqn.(42). Naturally, the state of $\mathrm{s}=0$ (or of $q=q_{\mathrm{o}}$ ) is occupied by almost all particles at $T_{\lambda}$; the state of the system at $T<T_{\lambda}$ differs only in the energy of $K$-motions which now corresponds to collective excitations of the system such as phonons and rotons. Consequently, using $q=q_{\mathrm{o}}$ for all particles in Eqn.21, the state of a SIB at $T \leq T_{\lambda}$ can be expressed by

$$
\begin{gathered}
\Phi_{\mathrm{n}} \mathrm{S}(N)=\Phi_{o}(N) \Psi_{K}(N) \text { with } \\
\left.\Psi_{K}(N)=\Sigma_{P}^{N !}( \pm 1)^{P} \prod_{i}^{N} \exp \left(i P \mathbf{K}_{i} \cdot \mathbf{R} i\right)\right]
\end{gathered}
$$

The superscript $\mathrm{S}$ in $\Phi_{n}{ }^{\mathrm{S}}(N)$ signifies the fact that the system becomes superfluid at $T \leq T_{\lambda}$ with all the $N$ ! micro-states $\left(\Psi_{n}^{j}(N)\right.$, given by Eqn.20) appearing in $\Phi_{n}{ }^{S}(N)$ (Eqn.21) merge into one state $\left(\Phi_{n}{ }^{\mathrm{S}}(N)\right.$; this implies that the entire system at $T \leq T_{\lambda}$ attains a kind of oneness as envisaged by Toubes[59].

\subsection{Volume Expansion on Cooling}

In what follows from Sections 4.1, 4.2 and 4.6, the $q$-motion energy of almost all particles gets frozen at $q=q_{\mathrm{o}}$ with corresponding energy $\varepsilon_{0}\left(T_{\lambda}\right)=h^{2} / 8 m d^{2}$ when a SIB is cooled to $T=T_{\lambda}$. However, if the system is cooled below $T_{\lambda}$, its each particle tries to, naturally, have an energy $<\varepsilon_{0}\left(T_{\lambda}\right)$ which, obviously, corresponds to $\lambda / 2>d_{\lambda}$ which does not agree with the restriction $\lambda / 2<d$ (Eqn.16). Evidently, any fall in $q$-motion energy below $\varepsilon_{0}\left(T_{\lambda}\right)$ needs an increase in $d$ beyond $d_{\lambda}$ (decided by a balance of forces representing $V^{\mathrm{R}}\left(r_{i j}\right)$ and $V^{\mathrm{A}}\left(r_{i j}\right)$ ); however, this is made possible by the zero-point repulsive force, $f_{\mathrm{o}}=-\partial_{d} \varepsilon_{\mathrm{o}}=h^{2} / 4 m d^{3}$, exerted by each particle on its neighbors against the inter-particle attraction, $f_{\mathrm{a}}$ (originating from $V^{\mathrm{A}}\left(r_{i j}\right)$ ). It is clear that $f_{\mathrm{o}}$ comes into operation effectively only around $T_{\lambda}$. We study the equilibrium of $f_{\mathrm{o}}$ and $f_{\mathrm{a}}$ in our recent study[60] of a quantum particle trapped in a 1-D box and Section 5.1(ii) for the present case. It is evident that the said equilibrium leaves an increase in the cavity size by $\Delta d=d_{T}-d_{\lambda}$ for almost all particles at $T_{\lambda}$. Consequently, the system on its cooling has to have a volume expansion around $T_{\lambda}$ rendering $\varepsilon_{\mathrm{o}}$ of each particle to decrease by

$$
\Delta \epsilon(T)=\left[\varepsilon_{0}(T)-\varepsilon_{0}\left(T_{\lambda}\right)\right]=-2 \varepsilon_{0}\left(\Delta d / d_{\lambda}\right)
$$

However, as shown in Section 5.1(ii) (as well as in[60]), the net fall in energy is only $(1 / 2) \Delta \epsilon(T)$ because $(1 / 2) \Delta \epsilon(T)$ energy is retained with the system as a share of each particle in the strain energy of inter-particle bonds which are forced to have an expansion by $\Delta d$. The fact that LHe-4 is found to exhibit volume expansion on its cooling through $\left.T_{\lambda[} 2(a)\right]$ not only proves the accuracy of this prediction of our theory but also provides strong experimental support to the WP manifestation of a particle (a well known consequence of wave particle duality) in relation to the behavior of particles in a SIB like LHe-4.

\subsection{Quantum Correlations and Their Importance}


Here we show that quantum correlations between particles, originating from their wave nature, play an important role in relation to the behavior of a SIB. These can be expressed in terms of what is known as quantum correlation potential (QCP)[61-63] obtained by comparing the partition function (under the quantum limits of the system), $Z q=$ $\Sigma_{\mathrm{n}} \exp \left(-E_{\mathrm{n}} / k_{B} T\right)\left|\Phi_{\mathrm{n}} \mathrm{S}^{\mathrm{S}}(N)\right|^{2}$ and its classical equivalent, $Z_{\mathrm{c}}=$ $\Sigma_{\mathrm{n}} \exp \left(-E_{\mathrm{n}} / k_{B} T\right) \exp \left(-U_{\mathrm{n}} / k_{B} T\right)$. Here $\Phi_{\mathrm{n}} \mathrm{S}^{\mathrm{S}}(N)$ is given by Eqn.52; note that the system at any given instant, even at $T>$ $T_{\lambda}$, exists in one of the $N$ ! micro-states $\Psi_{\mathrm{n}}^{j}(N)$ (Eqn.20) which hardly differs from $\Phi_{\mathrm{n}}{ }^{\mathrm{S}}(N)$ particularly at $T_{\lambda}$. The application of this method to our system is justified because our theory describes its particles by summarized plane waves rendering $\left\langle\mathrm{V}_{\mathrm{HC}}(r)\right\rangle=0$ which indicates that the $\mathrm{HC}$ potential is completely screened out. Simplifying $U_{\mathrm{n}}$, one easily finds that pair wise QCP has two components, viz.,

$U_{i j}^{\mathrm{S}}=-k_{B} T_{0} \ln \left[2 \sin ^{2}(\varphi / 2)\right]=-k_{B} T_{0} \ln \left[2 \sin ^{2}(2 \pi r / \lambda)\right] \quad(54)$

which pertains to the relative motion of two particles; here $\lambda=2 \pi / q$ is the de Broglie wavelength of a particle for its $q$-motion and $T_{\mathrm{o}}$ replaces $T$ because the $T$ equivalent of $q$-motion energy at all $T \leq T_{\lambda}$ is $T_{\mathrm{o}}$. It is evident that $U_{i j}^{\mathrm{S}}$ controls the relative $\varphi$-position of two nearest neighbor.

Following the approach of obtaining QCP for non interacting bosons[63], we easily find the second component

$$
U_{i j}=-k_{B} T \ln \left[1+\exp \left(-2 \pi\left|\mathbf{R}^{\prime}-\mathbf{R}^{\prime \prime}\right|^{2} / \lambda_{T}^{\prime 2}\right)\right]
$$

which pertains to $K$-motions of particles with $\lambda_{T}^{\prime}$ defined in Section 4.5.

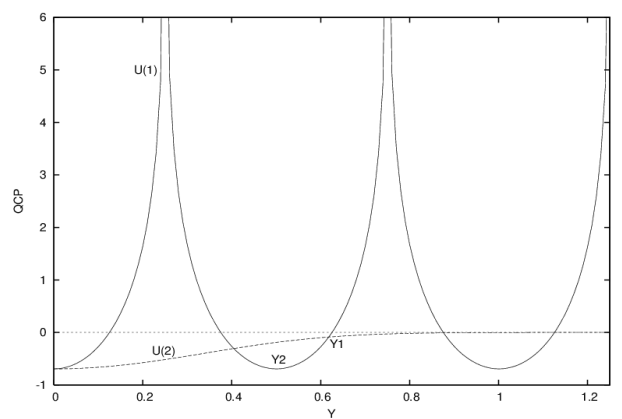

Figure 3. Quantum correlation potential (QCP) $U_{\mathrm{ij}}^{\mathrm{S}}$ (Eqn.54 traced as Curve $U(1)$ ) and $U_{i j}$ (Eqn.55 traced as Curve $U(2)$ ) plots against a dimensionless parameter $Y$ which represents: (i) $Y=Y_{\mathrm{q}}=r / \lambda$ for Eqn.54, and (ii) $Y$ $=Y_{\mathrm{K}}=\left|\mathbf{R}^{\prime}-\mathbf{R}^{\prime \prime}\right| / \lambda_{T}^{\prime}$ for Eqn.55 (See Section 4.8 for details)

We plot $U_{i j}^{\mathrm{S}}($ Curve $U(1))$ and $U_{i j}($ Curve $U(2))$ in Fig.3 against a dimensionless parameter $Y$ which represents: (i) $Y_{q}$ $=r / \lambda$ for Eqn.54 and (ii) $Y_{K}=\left|\mathbf{R}^{\prime}-\mathbf{R}^{\prime \prime}\right| / \lambda_{\mathrm{T}}^{\prime}$ for Eqn.55. We note that different particles in the system at $T \neq 0$ can be identified with pairs which may have different, $Y_{q} \geq 0.5$, -a condition derived from our inference $\lambda / 2 \leq d$ (Eqn.16) for $\mathrm{HC}$ particles. Accordingly, when the falling $T$ approaches $T_{\lambda}$, these $Y_{q}$ points converse on $Y_{q}=0.5$, since $\underline{U}_{i j}^{\mathrm{S}} / k_{B} T_{\mathrm{o}}$ has its minimum value $(-\ln 2)$ at this point. Evidently, particles in the system get locked at this point implying that they all have $\lambda / 2=d$ or $q=q_{\mathrm{o}}$. However, the same particles (for their $K$-motions), represented by different $Y_{K}$ points (for different $K$ between 0 and $\infty$ ) continue to move toward $Y_{\mathrm{K}}=0$ where $U_{i j} / K_{B} T$ has its minimum value $(-\ln 2)$ and this process ends only at $T=0$. In other words the condensation of particles in
$K=0$ state, which starts immediately after "all particles" for their $q$-motion get locked at $Y_{q}=0.5$, is completed at $T=0$.

Fig.3 also reveals that: (i) macro-orbital states become energetically favorable when $Y_{q}$ representing a particle or its representative pair assumes a value $<\mathrm{Y} 1$ where $U_{i j}{ }^{\mathrm{S}}<U_{i j}$, (ii) the $K$-motion of each particle gets delinked from $q$-motions as soon as particles are locked at $q=q_{\mathrm{o}}$ which leaves no possibility for any change in their $q$-value on further cooling of the system, (iii) the relative $r-$ and $\varphi-$ positions of particles are also locked, respectively, at $\langle r\rangle=\lambda / 2$ and $\Delta \varphi=2 \pi$ as soon as the system reaches at $Y_{q}=0.5$ and (iv) the system at $T \leq T_{\lambda}$ has only $K$-motions to contribute for its thermal energy because almost all particles have their lowest $q\left(=q_{\mathrm{o}}\right)$ at these temperatures.

Since $U_{i j}^{\mathrm{s}}$ and $U_{i j}$ are simple representatives of wave mechanical probabilities, not real potentials, the above analysis demonstrates the way the wave nature guides BEC of particles as (q, $-\mathbf{q})$ pairs in a state of $q=q_{\mathrm{o}}$ and $K=0$ and beautifully organizes them in a relative configuration defined by Eqn.29 through $U_{i j}^{\mathrm{S}}$ and $U_{i j}$ clubbed with zero point force (another consequence of wave nature) that keeps two particles at $r \geq \lambda / 2$.

\section{Superfluidity and Related Aspects}

\subsection{Energy Gap and Bound (q,-q) Pairs}

Following Sections 4.1 and 4.4, we note that: (i) cooling a SIB through $T_{\lambda}$ decreases $F(K)$ (Eqn.44) to its zero value at $T$ $=0$ and keeps $F(q)\left(\right.$ Eqn.43) at $F\left(q_{0}\right)=N \varepsilon_{0}$ which remains nearly constant for a change in $T$ from $T_{\lambda} \rightarrow T=0$, and (ii) $\rho_{\mathrm{s}}$ (representing the order parameter, $\Omega$, Section 4.4, Fig.2) has maximum value at $T=0$ where $K$-motions cease to exist. Evidently, the most relevant part of the free energy which is responsible for the superfluidity and related properties of LT phase of a SIB is $F(q)$, -not $F(K)$. This is corroborated by the fact that $\rho_{\mathrm{s}}$ decreases with increasing $T$ indicating that increase in the amount of thermal excitations (or their representative $F(K)$ ) has a counter effect on these properties.

In what follows from Section 4.7, each particle in a SIB exerts zero point force $f_{\mathrm{o}}$ which not only tries to keep two particles at a distance of $\lambda / 2$ (the size of their WPs) which increases with decreasing $T$ but also forces an increase in $\mathrm{d}$ against the inter-particle attraction $f_{\mathrm{a}}$. Since $f_{\mathrm{o}}$ comes into force before such a SIB reaches its $T$ of solidification, $V^{\mathrm{A}}\left(r_{i j}\right)$ fails to transform the fluid into solid as its normal goal. However, $V^{\mathrm{A}}\left(r_{i j}\right)$ limits the inevitable increase in $d$ (or equivalent increase in $\mathrm{V}$ ) to a smallest possible value $\Delta d=d_{\mathrm{T}}$ $-d_{\lambda}$. Evidently such a SIB is expected to have its volume expansion around $T_{\lambda}$ by an amount permitted by a balance between $f_{\mathrm{o}}$ and $f_{\mathrm{a}}$. The fact that this expectation agrees with experimentally observed volume expansion of LHe-4 on its cooling through $T_{\lambda}[2(\mathrm{a})]$ not only reveals that the onset of $\lambda$-transition also has its relation with the interplay of $f_{\mathrm{o}}$ and $f_{\mathrm{a}}$ but also provides experimental support to the WP manifestation of particles in the fluid at $T=0 \rightarrow T_{\lambda}{ }^{+}$(a $T$ little above $T_{\lambda}$ ); this is not surprising since the WP manifestation of a 
particle is one of the basic foundations of wave mechanics. Interestingly, as concluded from the following discussion, the balance of $f_{\mathrm{o}}$ and $f_{\mathrm{a}}$ also renders a kind of collective binding $E_{g}(T)$ (or an energy gap between S-state such as $\mathrm{He}-\mathrm{II}$ and N-state such as He-I) (see Eqn.60, below) among all the $\mathrm{N}$ particles below $\lambda$-point. Qualitatively, this kind of binding is also concluded[cf., point (i) below] by following a standard method used to understand molecular binding[64] where two identical atomic orbitals form two molecular orbitals of bonding and anti-bonding nature.

(i). $V^{\mathrm{A}}\left(r_{i j}\right)$ as source of collective binding: To understand how $V^{\mathrm{A}}\left(r_{i j}\right)$ produces binding of two particles like ${ }^{4} \mathrm{He}$ atoms in the state of their wave superposition, we diagonalize $(2 \times 2)$ energy matrix, defined by $E_{11}=E_{22}=\varepsilon_{0}$ and $E_{12}=E_{21}=\beta_{0}$, with $\beta_{\mathrm{o}}=\left\langle V^{\mathrm{A}}\left(r_{i j}\right)>\right.$; to this effect we note that two such particles have identically equal energy $\varepsilon_{\mathrm{o}}(c f$., Fig.1(middle)). The diagonolization renders two states of energy $\varepsilon_{0} \pm\left|\beta_{o}\right|$ for the pair; one may better replace $\left|\beta_{0}\right|$ by $\left|\beta_{0}(T)\right|$ since the overlap of macro-orbitals of two particles may depend on $T$. The states of energy $\left(\varepsilon_{0}-\left|\beta_{0}(T)\right|\right)$ and $\left(\varepsilon_{0}+\left|\beta_{0}(T)\right|\right)$ can, respectively, be identified as bonding (or paired) and anti-bonding (or unpaired) states. The pair is expected to be in bonding state ( $c f$., Fig.1(right)) provided the two particles remain locked in the relative configuration characterized by Eqn.29. The fact, that this situation arises for almost all particles (excluding a small fraction of particles in states of $q$ $\geq 2 q_{\mathrm{o}}$ ) only at $T \leq T_{\lambda}$, distinguishes the state of particles in the LT phase (such as He-II) from that in HT phase (He-I). In the following discussion we use bonding states of particles to conclude what we call as an energy gap $E_{\mathrm{g}}(T)$ (cf. Eqn.60, below) or their collective binding.

Applying the same approach to the state of all the $N$ particles, we construct a $N \mathrm{x} N$ matrix for $H_{r}(N)=-\left(\hbar^{2} / m\right) \Sigma_{\mathrm{i}} \nabla_{\mathrm{i}}^{2}+$ $\Sigma_{\mathrm{i}<j}\left[\mathrm{~A}\left(r_{i j}\right) \delta\left(r_{i j}\right)+\mathrm{V}^{\mathrm{A}}\left(r_{i j}\right)\right]$ (where we use Eqn.22 and retain terms only related to $q$-motions of particles) with $\left[H_{\mathrm{r}}(N)\right]_{\mathrm{mn}}=$ $\varepsilon_{\mathrm{o}}$ for $\mathrm{m}=\mathrm{n}$ and $\left[H_{\mathrm{r}}(N)\right]_{\mathrm{mn}}=[V(r)]_{\mathrm{mn}}$ for $\mathrm{m} \neq \mathrm{n}$ with $[V(r)]_{\mathrm{mn}}$ having non-zero value only if $\mathrm{m}$ and $\mathrm{n}$ refer to two neighboring particles. While, this requires, in principle, the knowledge of the number of neighbors of a particle (which depends on the symmetry of the spatial arrangement assumed by the particles), such details are not needed since quantitative value of energy gap $E_{g}(T)$ ( $c f$., Sub-section (ii) and Eqn.60, below) can be determined accurately by using the balance of $f_{\mathrm{o}}$ and $f_{\mathrm{a}}$. The diagonalization of the said matrix renders $N / 2$ energy levels, identified as anti-bonding states [as they have energies $\left.>N \varepsilon_{0}\left(T_{\lambda}\right)\right]$ and $N / 2$ energy levels, identified as bonding states[as they have energies $<N \varepsilon_{0}\left(T_{\lambda}\right)$ ]. Particles, obviously, prefer to occupy bonding states leading the net energy of the system to fall below $N \varepsilon_{0}\left(T_{\lambda}\right)$ by $E_{g}(T)$. Although, $2\left|\beta_{0}(T)\right|$ (the measure of binding of particles in a pair), may have very small value, however, as shown in[65, 66] the collective binding $\left(E_{g}(T)\right)$ of macroscopically large number of particles, resulting from it, is found to be good enough to counter thermal excitations. Naturally, $E_{g}(T)$ makes the S-state of a SIB stable against thermal motions and low energy perturbations such as the flow (linear or rotational) of the fluid with velocities below certain critical values.

(ii). Energy gap and balance of $f_{o}$ and $f_{a}$ : When a SIB has its volume expansion (as concluded in Section 4.7) due to zero point force coming into operation at $T_{\lambda}$, the sum of: (i) $h^{2} / 8 m\left(d_{\lambda}+x\right)^{2}$ representing the zero point energy of a particle and (ii) $(1 / 2) c x^{2}$ representing its share in the strain energy of expanded inter-particle bonds can be expressed by

$$
E(T, x)=(1 / 2) c x^{2}+h^{2} / 8 m\left(d_{\lambda}+x\right)^{2}
$$

where $x$ is an expansion (at $T \leq T_{\lambda}$ ) in $d_{\lambda}$. To this effect we ignore the $\mathrm{K}$-motion energy of the particle which remains unaffected by inter-particle interactions or their ramifications such as zero point force, $f_{\mathrm{o}}=-\partial_{x}\left[h^{2} / 8 m\left(d_{\lambda}+x\right)^{2}\right]$, -a consequence of $\mathrm{HC}$ repulsion clubbed with WP manifestation of particle. It may be noted that $(1 / 2) c x^{2}$ is nothing but a harmonic potential contributed by $V^{\mathrm{A}}\left(r_{i j}\right)$ and this renders $f_{\mathrm{a}}=$ $-\mathrm{c} x$ that opposes $f_{\mathrm{o}}$. Using

$$
\left.\partial_{x} E(T, x)\right|_{x=\Delta d}=0
$$

for the state in equilibrium between $f_{\mathrm{o}}$ and $f_{\mathrm{a}}$, at $x=\Delta d=$ $d_{\mathrm{T}}-d_{\lambda}$, we have,

$$
(1 / 2) \mathrm{c} \Delta d^{2}=h^{2} \Delta d / 8 m\left(d_{\lambda}+\Delta d\right)^{3}
$$

Using this relation with Eqn.(56) to separately find $E(T, x)$ for $x=\Delta \mathrm{d}$ as well as $x=0$ and then determine $\Delta E=E(T, \Delta d)$ $-E\left(T_{\lambda}, 0\right)$ given by

$$
\Delta E=-h^{2} \Delta d / 8 m d_{\lambda}^{3}
$$

We note that this equals half the value of $\Delta \epsilon(T)$ (Eqn.53) representing the net loss in zero point energy, $\varepsilon_{0}(T)-\varepsilon_{0}\left(T_{\lambda}\right)$, of a particle. This means that half of this loss is stored as strain energy in strained bonds, while the other half moves out of the system when it is cooled to a chosen $T$ starting from $T_{\lambda}$. Since this happens to all particles in the system, the net fall in energy of the particles can be expressed by

$$
E_{\mathrm{g}}(T)=-N|\Delta \epsilon(T)| / 2=-N \varepsilon_{\mathrm{o}}(T) \Delta d / 2 d
$$

In view of the fact that the onset of $\lambda$-transition delinks $q$-motions from $K$-motions for which $F(q)$ and $F(K)$ assume their separate identities, it becomes amply clear that to bring the superfluid SIB back to the configuration of its N-phase (viz., to transform He-II at a chosen $T$ ) to the configuration of He-I (at $T_{\lambda}{ }^{+}$), we need to supply $E_{\mathrm{g}}(T)$ energy from outside. This, evidently, means that $E_{\mathrm{g}}(T)$ represents an energy gap between S-phase and N-phase of a SIB.

(iii). Bound/unbound pairs in LT/HT phase: In view of the fact that each particle in a SIB is a part or a representative of a $(\mathbf{q},-\mathbf{q})$ pair even in HT phase close to $T_{\lambda}$, the zero and non zero value of $E_{\mathrm{g}}(T)$ in HT and LT phases, respectively, imply that the representative pair is an unbound $(\mathbf{q},-\mathbf{q})$ pair in HT phase, while the same in LT phase is a bound (q, -q) pair. The energy levels of these pair states are, respectively, depicted in Fig.1(middle) and Fig.1(right) for their comparison and better perception. The figure also shows that two particles, which occupy levels of different energies $\left(E_{1}\right.$ and $\left.E_{2}\right)$ in the absence of their wave superposition (i.e., $\lambda / 2<<d$ ) assume a single quantum level of a unbound pair (where each particle share $E_{1}+E_{2}$ energy equally) when they happened to have their wave superposition for their $\lambda / 2 \approx d$ at a $T \approx T_{\lambda}$. While this indicates that wave superposition pushes two particles of different energies into a single state of energy $\left(E_{1}\right.$ $\left.+E_{2}\right) / 2$, the argument behind Eqn.(31) indicates that two 
particles in the $N$-state of a SIB, particularly, at $T$ close to $T_{\lambda}$ can be assumed to have $2 \varepsilon_{0}$ energy. Further, since $E_{\mathrm{g}}(T)$ is the net binding energy of all the $N$ particles, the entire system at $T<T_{\lambda}$ behaves like a macroscopically large single molecule as envisaged by Foot and Steane[67] for the BEC state of TDG. Evidently, our theory predicts that the stability of S-state of a SIB cannot be disturbed by any perturbation of energy $<E_{\mathrm{g}}(T)$.

(iv). New quantum quasi-particle (OMON) in LT phase: Each particle in LT phase of the system has $\epsilon_{\mathrm{S}}(T)=\Delta \epsilon(T) / 2$ energy as its share in the net strain energy $\Delta V_{\mathrm{S}}(T)=N \epsilon_{\mathrm{S}}(T)$ of the expanded inter-particle bonds ( $c f$., Section. 4.7 and Eqn.53); we propose to call $\epsilon_{\mathrm{S}}(T)$ as the self energy of a particle. Evidently, particles in the LT phase of the system are pushed to higher potential energy in comparison to those in HT phase and this increase depends on the WP size $\lambda / 2(=$ $\pi / q)$ of the particles. This means that $\Delta V \mathrm{~s}(T)$ is a function of $q$ values of particles i.e., $\Delta V \mathrm{~s}=\Delta V_{\mathrm{s}}\left(q_{1}, q_{2}, \ldots q_{N}\right)$ which, obviously, prepares the system to sustain phonon like waves of collective oscillations of momentum $q$ around $q_{\mathrm{o}}$ which could be identified as a new quantum quasi-particle proposed to be known as omon. The existence of these waves is predicted for the first time from the present theory. A detailed discussion on omons is available in[65] which shows that energy dispersion for omons does not differ from that of phonons in the system. Identifying $\Delta V \mathrm{~s}(\mathrm{~T})\left(q_{1}, q_{2}, q_{3}, ..\right)$ as the energy of omon field and using the fact that $\Delta V \mathrm{~s}(T)$ increases with decreasing $T$ (which implies that omon field intensity increases when phonon field intensity decreases and vice versa), it may be concluded that an omon is an anti-phonon quantum quasi-particle. Evidently, collective motions, such as phonons, can be seen in the system even at $T=0$ at cost of omon field energy $\Delta V \mathrm{~s}(T)$ which assumes maximum value at $T=0$. As discussed in Section 6.2(1), $\Delta V \mathrm{~s}(T)$ also serves as the origin of a force for which S-fluid component flows from lower to higher $T$.

(v). Difference with Cooper pair of electrons: In the light of Eqn.29, the binding of particles in the LT phase of a SIB clearly means that their positions are energetically locked at $\langle k\rangle=0,\langle r\rangle=d$ and $\langle\varphi\rangle=2 n \pi$ with per particle binding energy, $E_{\mathrm{g}}(T) / N$, in all the three $(k-, r-$ and $\varphi-)$ spaces. We call it collective binding in a sense that each particle has its binding with all the $(N-1)$ particles in the system. For all these reasons, the $(\mathbf{q},-\mathbf{q})$ bound pair in our system differs from $(\mathbf{q},-\mathbf{q})$ bound pair of electrons (known as Cooper pair used in the BCS theory of metallic superconductors[34, 68]). While the binding of electrons in a Cooper pair originates from electrical strain (polarization) of the lattice, binding of atoms in a SIB is an inter-play of $f_{\mathrm{o}}$ and $f_{\mathrm{a}}$ which leads to an expansion of all the inter-atomic bonds (a kind of mechanical strain in the system). In addition, while bound pair formation in a superconductor includes only those electrons which occupy energy levels around the Fermi-level of the system, bound pair formation in a SIB arrests all the $\mathrm{N}$ bosons.

\subsection{Energy Gap and its Consequences}

In what follows from Section-4.4, a SIB has two compo- nents, fluid $\mathrm{F} 1$ and fluid $\mathrm{F} 2$ with free energies represented, respectively, by $F(K)$ and $F(q) . F(K)$ and $F(q)$ get delinked from each other at $T_{\lambda}$ with freezing of $q$-motion at $q=q_{\mathrm{o}}(c f$. Section 4.8). Hence the fact, that $F(K)$ represents quasi-particle excitations originating from $K$-motions (unaffected by interactions), implies that only $F(q)$ is affected by $E_{\mathrm{g}}(T)$ and we have $F(q)=N \varepsilon_{\mathrm{o}}\left(T_{\lambda}\right)-E_{\mathrm{g}}(T)$ which, obviously, means that the origin of different LT properties (including superfluidity and related aspects) of a SIB lies with $E_{\mathrm{g}}(T)$. We use this inference to analyze the following aspects of a SIB at $\mathrm{T} \leq \mathrm{T}_{\lambda}$.

\subsubsection{Superfluidity and Related Properties}

If two heads $\mathrm{X}$ and $\mathrm{Y}$ in the system have small $T$ and $P$ (pressure) differences, the equation of state can be expressed as $E_{\mathrm{g}}(\mathrm{X})=E_{\mathrm{g}}(\mathrm{Y})+S \Delta T-\mathrm{V} \Delta P$. Using $E_{\mathrm{g}}(\mathrm{X})=E_{\mathrm{g}}(\mathrm{Y})$ for equilibrium, we get

$$
S \Delta T=\mathrm{V} \Delta P
$$

This reveals that: (i) the system should exhibit thermo-mechanical and mechano-caloric effects and (ii) while, the measurement of $\eta$ by capillary flow method performed under the condition $\Delta T=0$ should find $\eta=0$, measurement of thermal conductivity $(\Theta)$ under $\Delta P=0$ should find $\Theta \approx \infty$. All these predictions are found to be true with experimentally observed behavior of LT phase of LHe-4. He-II is a well known superfluid of infinitely high $\Theta$.

Interestingly, several important aspects of our system can also be followed qualitatively from the configuration of F2 defined by Eqn.29. For example (i) a CPA-WP in a fluid like system can have no vacant site, particularly, because two neighboring particles experience zero point repulsion which, naturally, means that the system should have very large $\Theta$ which further implies that the system cannot have temperature gradient and convection currents and this explains why He-II does not boil like He-I, (ii) since particles in F2 cease to have relative motion and they can move only in order of their locations, the system is bound to exhibit vanishingly small $\eta$, particularly, for their linear flow in narrow capillary, etc.; however, the rotating fluid may exhibit normal viscous nature since particles moving on the neighboring concentric circular paths of quantized vortices have relative velocity which explains both viscosity and rotation paradoxes[5]. In what follows from these observations the loss of viscosity in linear flow is not due to any loss of viscous forces among the particles, rather it is the property of the LT phase configuration in which particles cease to have relative or collisional motion.

\subsubsection{Critical Velocities and Stability of LT phase}

Following Eqn.50 and the basic arguments behind it, we find that the G-state wave function $\Phi_{\mathrm{o}}(N)$ changes to

$$
\begin{aligned}
& \Phi^{*}(N)=\Phi_{\mathrm{o}}(N) \exp \left(i \mathbf{K} \cdot \Sigma^{\mathrm{N}} \mathbf{R} i\right) \\
& \exp \left[-i\left[N\left(\varepsilon_{\mathrm{o}}+\varepsilon(K)\right)-E_{\mathrm{g}}(T)\right] \mathrm{t} / \mathrm{h}\right.
\end{aligned}
$$

when the system is made to flow with velocity $v_{\mathrm{f}}=\hbar \Delta q / \mathrm{m}$. This reveals that $\Phi_{0}(N)$ remains stable against the flow unless its energy, $N m v_{\mathrm{f}}^{2} / 2=N \varepsilon(K)$, overtakes the collective binding $E_{\mathrm{g}}(T)$ and this fact should explain critical velocity $v_{\mathrm{c}}$ 
for which the system loses its superfluidity totally. Equating $E_{\mathrm{g}}(T)$ and $N m v_{\mathrm{f}}^{2} / 2$ with $v_{\mathrm{f}}=v_{\mathrm{c}}$, we obtain

$$
v_{\mathrm{c}}(T)=\sqrt{ }\left(2 E_{\mathrm{g}}(T) / N m\right)
$$

which represents its upper bound. A $v_{\mathrm{c}}<v_{\mathrm{c}}(T)$, at which a superfluid may show signs of viscous behavior, can be expected due to creation of quantized vortices. However, this cause would not destroy superfluidity unless energy of all vortices produced in the system exceeds $E_{\mathrm{g}}(T)$.

\subsubsection{Coherence length}

Since the main factor responsible for the coherence of F2 is its configuration which locks the particles at $\Delta \varphi=2 n \pi$ (cf. Eqn.29) with collective binding $E_{\mathrm{g}}(T)$, the coherence length (not to be confused with healing length[5]) can be obtained from

$$
\xi(T)=h / m v_{\mathrm{c}}(T)=h\left[N / 2 m E_{\mathrm{g}}(T)\right]=2 d(d / \Delta d)
$$

\subsubsection{Superfluid Density}

Correlating the superfluid density, $\rho_{\mathrm{s}}$, as the order parameter of the transition, with $E_{\mathrm{g}}(T)$, we find

$$
\rho_{\mathrm{s}}(T)=\left[E_{\mathrm{g}}(T) / E_{\mathrm{g}}(0)\right] \rho(T)=\left[\left(d_{\mathrm{T}}-d_{\lambda}\right) /\left(d_{0}-d_{\lambda}\right)\right] \rho(T)
$$

to determine $\rho_{\mathrm{s}}(T)$ and normal density, $\rho_{\mathrm{n}}(T)=\rho(T)-\rho_{\mathrm{s}}(T)$. Evidently, $v_{\mathrm{c}}(T), \xi(T)$, and $\rho_{\mathrm{s}}(T)$ can be obtained if we know $E_{\mathrm{g}}(T)$ (Eqn. 60). Further since the S-state wave function (Eqn.52) is expected to vanish at the boundaries of the system, it is natural that $E_{\mathrm{g}}(T)$ and $\rho_{\mathrm{s}}(T)$ also vanish there.

\subsubsection{Superfluid Velocity}

Concentrating only on the time independent part of $\Phi_{\mathrm{o}}{ }^{*}(\mathrm{~N})$ (Eqn.62), we can have

$$
\begin{aligned}
& \text { By using } \quad \Phi_{\mathrm{o}}{ }^{*}(N)=\Phi_{\mathrm{o}}(N) \exp (\mathrm{i} S(R)) \\
& \quad S(R)=\mathbf{K} .\left(\Sigma_{\mathrm{i}}^{\mathrm{N}} \mathbf{R}_{\mathrm{i}}\right) \\
& \text { as the phase of the S-state. This renders } \\
& \qquad v_{\mathrm{s}}=(\hbar / 2 m) \nabla_{\mathrm{Rj}} S(R)=(\hbar / m) \Delta q
\end{aligned}
$$

as a relation for the superfluid velocity; here we use the fact that $\nabla_{\mathrm{Rj}} S(R)$ renders the momentum of the pair (not of a single particle). One may find that Eqn.66 does not differ from the superfluid wave function presumed in the $\Psi$-theory of superfluidity[37] (cf. Section (6.2(2) below) of Reference[34]); of course for the well defined phenomenological reasons, $\Psi$-theory assumes $S(R)$ to be a complex quantity.

\subsubsection{Quantized Vortices}

Using the symmetry property of a state of bosonic system, Feynman $[15,69]$ showed that $\kappa$ (the circulation of the velocity field) should be quantized and be given by $\kappa=n(\mathrm{~h} / \mathrm{m})$ with $n=1,2,3, \ldots$ However, Wilks[2(a)] has rightly pointed out that this account does not explain the fact that He-I to which Feynman's argument applies equally well, does not exhibit quantized vortices. Using Eqn.67, we find that

$$
\left.\kappa=\Sigma_{i} \mathbf{v}_{\mathbf{s}}(i)\right) . \Delta \mathbf{r}_{i}=(\hbar / m) \Sigma_{i} \Delta \mathbf{q}_{i} \cdot \Delta \mathbf{r}_{i}=n(h / m)
$$

by using the condition that $\Sigma_{\mathrm{i}} \Delta \mathbf{q}_{i} \cdot \Delta \mathbf{r}_{i}=2 n \pi$ which presumes that particles moving on a closed path maintain phase correlation. To this effect our theory reveals that particles have their $\varphi$-positions locked at $\Delta \varphi=2 n \pi$ only in the LT phase for which only this phase can exhibit quantized vortices. Since particles in HT phase have random distribution $(\Delta \varphi \geq 2 n \pi)$ in $\varphi$-space, they cannot sustain $\varphi$-correlation and quantized vortices

\subsection{Laser Like State}

We note that the system below $T_{\lambda}$ defines a 3-D network of SMWs, extending from its one end to another end without any discontinuity. In lasers too these are the standing waves of electromagnetic field that modulate the probability of finding a photon at a chosen phase point. The basic difference between the two lies in the number of bosons in a single anti-nodal region of a SMW. In case of lasers this could be any number since photons are non-interacting particles but for a SIB like ${ }^{4} \mathrm{He}$ or ${ }^{87} \mathrm{Rb}$ one such region can have only one atom.

\section{Facts which Corroborate Our Theory}

\subsection{Thermodynamic and Hydrodynamic Properties}

We note that the excitation spectrum of a $\operatorname{SIB}(E(Q)=$ $\hbar^{2} Q^{2} / 4 m S(Q)$, with $S(Q)$ being the structure factor of the fluid at wave vector $Q$ ), as concluded by our theory[70], not only agrees well with Landau spectrum[6(b)] at qualitative level but also matches closely with the experimentally observed spectrum[4, 25, 31] for LHe-4 at quantitative level. Similarly, the calculated values of $v_{\mathrm{c}}, \rho_{\mathrm{s}}$ and $\rho_{\mathrm{n}}$, etc., by using relevant relations (Section 5.2), are found to agree[71] closely with their experimental values for LHe-4[5]. In what follows these facts ensure that our theory has great potential to accurately account for the thermodynamic and hydrodynamic properties (including superfluidity and related aspects) of the S-phase of a SIB.

\subsection{Phenomenological Pictures}

\subsubsection{Two Fluid Theory}

As concluded from Sections 4.4 and 5.2, a SIB at $T \leq T_{\lambda}$ gets separated into two fluids F1 and F2. While, F2 has all characteristic properties of a superfluid viz., zero entropy, zero viscosity, etc., since it comprises particles in their G-state with their positions locked at $\langle k\rangle=0,\langle r\rangle=\lambda / 2=d$ and $\Delta \varphi=2 n \pi$ (Eqn.29) for which they cease to have relative motion (or collisional motion), F1 identified with a gas of non-interacting quasi-particle excitations, has all properties (such as non-zero entropy, non-zero viscosity, etc.) of a $\mathrm{N}$-fluid. This not only concludes that a SIB below $T_{\lambda}$ should, undoubtedly, behave as a homogeneous mixture of two fluids that have all properties envisaged by Landau[6] but also provides microscopic foundation for two fluid phenomenology. It further reveals that each particle with its $q$-motion participates in F2 and with $K$-motion in F1. It does not support the perception that certain atoms participate in F1 and rest in F2.

As an important aspect of Landau's two fluid theory, S-fluid component of He-II is assumed to flow in a direction 
opposite to $\mathrm{N}$-fluid component which flows from the point of higher $T$ to that of lower $T$. Our theory finds a sound reason for it. Since $\rho_{\mathrm{s}}(T)$ and $V_{\mathrm{S}}(T)=N \epsilon_{\mathrm{S}}(T)$ both have monotonic increase with decrease in $T$, a point of lower $T$ has higher amount of both, $\rho_{\mathrm{s}}(T)$ and $V_{\mathrm{S}}(T)$, in comparison with a point of higher $T$. Naturally, particles having higher value of $V_{\mathrm{S}}(T)$ at a region of lower $T$ experience a force which make them move towards the region of higher $\mathrm{T}$ where they have lower $V_{\mathrm{S}}(T)$ unless the two regions attain a state of equal $V_{\mathrm{S}}(T)$, i.e. a state of equal strain energy which happens to occur at points of equal $T$. Alternatively, this means that $\rho_{\mathrm{s}}(T)$ flows from the region of lower $T$ towards the region of higher $T$ unless the two regions have equal $\rho_{\mathrm{s}}(T)$. Note that liquids cannot sustain pockets of different strain energy, $N \epsilon_{\mathrm{S}}(T)$, obviously, for its fluidity which allows them to flow from the regions of higher strain energy to those of lower strain energy and this task is completed by flow of omons which have higher density in the regions of lower $\mathrm{T}$ in comparison to those of higher $T$.

\subsection{2. $\Psi$-theory}

We find that superfluidity of a SIB is basically a property of its F2 component (or the G-state) represented by $\Phi_{0}(N)$ (Eqn.28) which can also be expressed as

$$
\Phi_{\mathrm{o}}(N)=\sqrt{ } \mathrm{n}
$$

with $\mathrm{n}=N / \mathrm{V}$. However, when $\mathrm{F} 2$ is made to flow, its state is given by $\Phi_{\mathrm{o}}{ }^{*}(N)$ (Eqn.66). For the phenomenological basis that $\rho_{\mathrm{s}}(T)=\mathrm{n}_{\mathrm{s}} m$ (at $\left.0<T<T_{\lambda}\right)$ is $<\rho=\mathrm{n} m$, it is imperative to assume that the phase $S(R)$ (Eqn.67) is a complex quantity

$$
S(R)=\xi_{\mathrm{r}}(R)+i \xi_{\mathrm{i}}(R)
$$

this shows that our theory also renders microscopic foundation to $\Psi-$ theory [37].

\subsubsection{Quantum Phase Transition}

As discussed in Section 4.8, our theory finds that $\lambda$-transition and superfluidity are the results of quantum nature of particles. When this observation is clubbed with the fact that superfluidity is basically a property of F2 component (comprising particles in their $T=0$ state) of a SIB. However, F2 exists even at non-zero $T<T_{\lambda}$ due to its proximity with F1 (a gas of quasi-particle excitations) whose energy measures the $T$ of the system. In what follows $\lambda$-transition could be identified as a quantum transition[72] which occurs at non-zero $T$ for the said proximity. Although, particles in excited states attributed to their $q$-motions (viz., with $q=2 q_{\mathrm{o}}, 3 q_{\mathrm{o}}, \ldots$, etc.) are, naturally, the part of F2, however, their number are found to be only $\approx 5 \%$ at $T_{\lambda}$ and it decreases exponentially to 0 value at $T=0$.

\subsection{Other Experimental Observations}

(i). Physical reality of existence of an electron bubble: An excess electron in liquid helium exclusively occupies a self created spherical cavity (known as electron bubble) of certain radius, when it assumes its lowest possible energy in the cavity. To create the said cavity it exerts its zero-point force on the surrounding atoms against the forces originating from inter-atomic interactions and external pressure on the liq- uid[73]. It is evident that the bubble formation is a consequence of the facts that: (i) an excess electron experiences a strong short range repulsion with $\mathrm{He}$ atoms which does not allow its binding with the said atoms and (ii) the electron, for its quantum nature, manifests as a WP whose size increases with the decrease in its energy. This implies that any quantum particle that experiences similar repulsion with He atoms should have similar state in liquid helium and this is found to be true with positron[74] and other particles (ions, atoms, molecules, etc.[75]). Guided by these observations, it is natural to believe that each ${ }^{4} \mathrm{He}$-atom in LHe-4 should assume similar state when it occupies its lowest possible energy because it too is a quantum particle and it experiences strong short range repulsion with other helium atoms. Here we also find that the electron in a drifting bubble has two motions ( $q$ - and $K$-motions), respectively, identified with its zero-point motion as a trapped particle and its drift with the bubble. This not only helps in having a better understanding of the two motions of a single quantum particle in a SIB like LHe-4, but also proves that the state of the electron in electron bubble is not different from the state that we represent by a macro-orbital. In other words the existence of an electron bubble not only renders a clear experimental proof for the macro-orbital state of a HC boson in a SIB like LHe-4 and provides strong experimental foundation to our theory but also reveals that $\zeta(\mathrm{r})$ part of the macro-orbital (Eqn.(18)) for $\mathrm{s}^{-}, \mathrm{p}^{-}, \mathrm{d}^{-}$.... states can be represented to a good approximation by the respective state functions of a particle trapped in a spherical cavity of size $d[76]$.

(ii) Spectroscopy of molecules embedded in He-droplets or clusters: Experimental study of high resolution rovibrational spectra of embedded molecules (e.g., OCS $/ \mathrm{N}_{2} \mathrm{O}$ molecule in ${ }^{4} \mathrm{He}$ droplets and $4 \mathrm{He}_{\mathrm{y}}: \mathrm{OCS}$ or $4 \mathrm{He}_{\mathrm{y}}: \mathrm{N}_{2} \mathrm{O}$ clusters[77] where $\mathrm{y}=$ number of ${ }^{4} \mathrm{He}$ atoms) provides another foundation to our theory because these studies conclude that superfluidity exists even in systems having few ${ }^{4} \mathrm{He}$ atoms (viz., about 6 or more) which implies that the phenomenon has no relation with $p=0$ condensate since ${ }^{4} \mathrm{He}$ atoms in these systems are confined to a space of size, $s \approx 5 \AA$ for which each atom is expected to have reasonably high momentum $\approx \pi / s$ rather than zero. Further since each cluster is expected to have certain stable structure (which of course would depend on inter-particle interactions and may change with change in y), the embedded molecule sees a time independent potential which implies that ${ }^{4} \mathrm{He}$ atoms around the rotor cease to have collisional motions. In other words ${ }^{4} \mathrm{He}$ atoms in these droplets and clusters are localized with position uncertainty decided by their least possible momentum of their confinement which agrees closely with our theory. In addition, as inferred in Section 3.3, particles in S-state of a SIB can move with equal velocity on a closed path without any change in order of their locations which implies that particles on two such nearest possible paths (viz., two concentric circular paths) can have different velocities consistent with the theory of experimentally observed quantized circulation in superfluid ${ }^{4} \mathrm{He}[5]$. Evidently, it is not surprising that a molecule (or its cluster with few neighboring atoms 
which bind with it to follow its rotation) is observed to have free rotation within a 3-D shell or a ring (formed by ${ }^{4} \mathrm{He}$ atoms in its surrounding) that does not participate in the rotation. It also finds that $\mathrm{OCS}$ or $\mathrm{N}_{2} \mathrm{O}$ molecule embedded in S-state of ${ }^{4} \mathrm{He}$ droplets shows sharp rotational lines because ${ }^{4} \mathrm{He}$ atoms cease to have collisional motion but not in $\mathrm{N}$-state of ${ }^{3} \mathrm{He}[77(\mathrm{a})]$ where particles are known to have mutual collisions. In other words rotational lines do not have collisional broadening in superfluid ${ }^{4} \mathrm{He}$ which they have in liquid ${ }^{3} \mathrm{He}$. As such experimental observations related to the rovibrational spectra of embedded molecules support our theory.

\subsection{Experimental Evidence for CPA-WP}

Diffraction experiments using X-ray, neutron and electron beams are expected to provide accurate information about the atomic arrangement in any system provided the high energy/momentum of these radiations does not damage/perturb this arrangement. Although, atoms in CPA-WP cease to have their relative motions, they remain the parts and parcel of a fluid where shear forces have vanishingly small magnitude which makes CPA-WP highly fragile. It differs significantly from the atomic arrangement in a solid/crystal which have appreciably strong shear forces. Particles in CPA-WP remain free to move coherently (all with same velocity keeping their relative positions, residual momentum and the relative phase positions fixed) in order of their locations on a line/closed path (or bundles of parallel lines/closed paths). In other words, atoms in He-II can slip on a line/plane with respect to those on neighboring lines/planes; they are likely to get displaced from their positions with a collective motion when they are hit by particles of high energy/momentum in a beam of said radiations. Even the relative distance of particles is expected to change, since it depends on the size of their WPs $(\lambda / 2=\pi / q)$ which can have large fluctuations when the system is exposed to the said radiations. Evidently, the said diffraction tools are not suitable to get reliable information about the CPA-WP and it is for this reason that CPA-WP could not be detected for so long.

However, we identify other experiments which prove CPA-WP of ${ }^{4} \mathrm{He}$-atoms in He-II from the fact that this arrangement locks atoms at a distance $d$, relative momentum at $k=2 q_{\mathrm{o}}=2 \pi / d$ and relative phase position $\Delta \varphi=2 n \pi$. To this effect we note the following:

(1).The excitation spectrum $E(Q)$ of He-II matches closely with that predicted for a mono-atomic chain with atoms separated by d not only at low $Q$ (observed for most liquids) but also at high $Q(>2 \pi / d)$. The momentum and energy of an excitation at such a high $Q$ basically correspond to a motion of a single particle for the fact that the corresponding wave length $\Lambda<d$ (the size of space occupied by a particle); this clearly proves that particles have an orderly arrangement with a separation $d$.

(2) Landau two fluid model which explains the properties of He-II to a good accuracy envisages S-component of He-II to have $S=0$ and $\eta=0$ and CPA-WP representing this component has $S=0$ because all particles occupy single quantum state of $q=q_{\mathrm{o}}$ and $\eta=0$ because particles cease to have relative motions or mutual collisions for their relative positions remain locked with $r=d$.

(3) Superfluid is observed to have coherent motion and vortices of quantum circulation and their possibility demands a configuration like CPA-WP where particles satisfy $\Delta \varphi=2 n \pi$.

Although, the above listed experimental observations have been there for many years, however, these were not analyzed to see the existence of CPA-WP because it was not perceived in the framework of CMT. Finally, we also note that several other experimental observations on He-II, such as $T^{3}$ dependence of specific heat, infinitely high thermal conductivity, etc. too support CPA-WP.

\section{Comparison of our NCMT and CMT}

\subsection{Important Aspects of our NCMT}

Our approach to the microscopic understanding of a SIB uses PPB which identifies each particle as a representative or a part of a pair of particles moving with equal and opposite momenta (q, $-\mathbf{q})$ with respect to their $\mathrm{CM}$ which moves with momentum $\mathbf{K}$. Consequently, the state of the particle is represented by a macro-orbital (Eqn.18) and the accuracy of this representation is established, beyond doubt, by the existence of electron bubble[Section 6.3(i)]. It is further strengthened by the fact that this representation identifies each particle to have two motions ( $q$ - and $K^{-}$) which provide the origin of two fluid behavior of He-II (Section 6.2(ii)).

The onset of $\lambda$-transition is an order-disorder of particles in $\varphi$-space followed, simultaneously, by their BEC in the state of $q=q_{\mathrm{o}}$ and $K=0$. The $T$ dependence of the condensate fraction $n_{K=0}\left(T^{*}\right)$ (Eqn. 49) starting from $n_{K=0}\left(T_{\lambda}\right)=0$ to $n_{K=0}\left(T^{*}=0\right)=1.0$ agrees closely with experimentally observed $\rho_{\mathrm{s}}(T) / \rho$ for He-II which represents a kind of order parameter of the transition (Curves $\mathrm{A}^{*}$ and E2, cf. Fig. 2).

The fact that $\lambda$-transition is a consequence of the wave nature of particles is made more clearly visible by our theory, since it finds: (i) how wave nature of particles leads to their quantum correlations represented by corresponding potentials $\left(U_{i j}^{\mathrm{S}}\right.$ (Eqn.54) and $U_{i j}($ Eqn.55) depicted in Fig. 3) which drive their $q$ and $K$, respectively, toward $q=q_{\mathrm{o}}$ and $K=0$, (ii) how zero-point force (another consequence of the wave nature), $f_{\mathrm{o}}=h^{2} / 4 m d^{3}$, exerted by each particle on its neighbors pushes them away against the inter-particle attraction, $f_{\mathrm{a}}$ (originating from inter-particle potential) and renders an increase in $d$ by $d_{T}-d_{\lambda}$ (Section 4.7) representing a kind of mechanical strain in inter-particle bonds when $f_{\mathrm{o}}$ and $f_{\mathrm{a}}$ reach a state of equilibrium, (iii) why energy of this strain, $\Delta V_{\mathrm{S}}(T)=N \epsilon_{\mathrm{S}}(T)$ (Section 5.1), depends on the $q$-values (fluctuating around $q_{\mathrm{o}}$ ) of particles for which the system sustains a new quantum quasi-particle omon similar to a phonon (Section 5.1), (iv) how zero-point energy (yet another consequence of wave nature) of each particle falls by small amount (Eqn.53) and how $E_{g}(T)$ (Eqn. 60) represents a 
collective binding between all atoms for which the entire system behaves like a macroscopic single molecule, (v) how collective binding serves as an energy gap $E_{g}(T)$ between S-state and N-state (Section 5.1), (vi) how this gap helps in accounting for the superfluidity and related properties of He-II (Section 5.2), and (vii) how zero-point force (Section 4.7) serves as a basis for our prediction that a SIB should exhibit-ve thermal expansion coefficient around $T_{\lambda}$ which is confirmed by the experimental observation on LHe-4[2(a)].

The theory reveals that the S-state is characterized by a fraction of particles $\left[n_{\mathrm{K}=0}\left(T^{*}\right)\right]$ condensed into the G-state represented by $q=q_{\mathrm{o}}$ and $K=0$ with no particle having $q<$ $q_{\mathrm{o}}$. It not only concludes the absence of $p=0$ condensate but also explains why existence of $p=0$ condensate in He-II could not be confirmed through any experiment beyond a point of doubt[26-28]. Inferences of our theory are also consistent with the criteria of the occurrence of BEC, viz. (i) the expected observation of ODLRO[55, 56], (ii) spontaneous symmetry breaking[57] and phase coherence[58], as well as with the excluded volume condition envisaged by Kleban[78] who argued that each ${ }^{4} \mathrm{He}-$ atom in LHe-4 occupies certain volume exclusively.

The theory also explains why LT phase of a SIB should behave like a homogeneous mixture of two fluids[(F1 (normal) and F2 (super) found to have different properties[7] as envisaged exactly by Landau's theory[6]] and thereby renders microscopic basis to two fluid theory and $\Psi$-theory[37]. It further reveals that Superfluidity and related properties are, basically, associated with the $T=0$ state of the system (represented by F2) which means that $\lambda$-transition is a kind of quantum transition which occurs at a non-zero $T$ for the proximity of F2 with F1.

Particles in F2 represent $(\mathbf{q},-\mathbf{q})$ bound pairs, not only in $q-$ space but also in $r$-and $\varphi$-spaces. They constitute a kind of CPA-WP and define a 3-D network of SMWs which locks their relative momentum, relative separation and relative position on $\varphi$-line, respectively, at $k=2 \pi / d, r=d$ and $\Delta \varphi=$ $2 n \pi$ with a net amount of collective binding represented by $E_{\mathrm{g}}(T)$; this provides reasons for the stability of S-state for small energy perturbations such as its flow (linear/rotational) with a velocity below certain values or small amplitude thermal oscillations. The S-state is consistent with microscopic uncertainty as evident from $q \geq \pi / d$ as well as macroscopic uncertainty since the 3-D network of SMWs represented by $\Phi_{0}^{\mathrm{S}}(N)$ (Eqn.28) vanishes at the boundaries of the system.

Our theory predicts that a SIB can have an onset of superfluidity provided it keeps its fluidity up to a $T \approx T_{\mathrm{o}} \equiv \varepsilon_{\mathrm{o}}$ ) at which the zero-point force of its particles dominates its physical state. Since the WP size $(\lambda / 2)$ for all particles in the S-state of a SIB remains as small as $d$ (Eqn.(42)), it is not surprising that superfluidity is observed in nano droplets and clusters of few atoms as reported recently in[75, 77].

The merit of our approach lies with the fact that it concludes the present theory simply by analyzing the solutions of its $N$-particle Schrodinger equation. It makes no assumption such as the existence of $p=0$ condensate or pair con- densate, etc. in the S-phase of a SIB as made by CMT. The theory is mathematically simple and its results cannot be manipulated since it uses no adjustable parameter. It does not use any drastic approximation in determining the role of inter-particle interactions. It beautifully demonstrates that the wave nature has amazing capacity to organize particles in phase space at $\varphi=2 n \pi$ with $\langle r\rangle=\lambda / 2=d$.

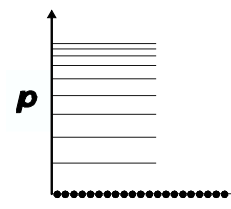

(A)

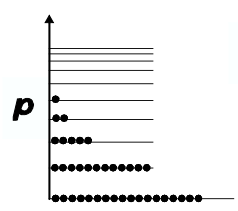

(B)

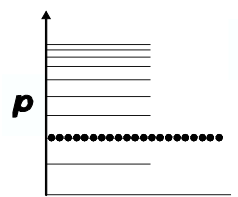

(C)

Figure 4. Schematic of distribution of particles at $T=0$. (A) All the $N$ particles occupy single particle state of $p=0$ in a system of non-interacting bosons, (B) depletion of $p=0$ condensate (i.e. only a fraction of $N$ occupy $p$ $=0$ state) in weakly interacting boson system as predicted by Bogoliubov model[8], and (C) all the $N$ particles occupy a state of $q_{\mathrm{o}}=\pi / d$ and $K=0$ as concluded from the present theory

\subsection{Important Aspects of CMT}

Following the basic theory of BEC[9] in SNIB, one finds that $100 \%$ particles occupy a single particle state of $p=0$ (presumed to represent the G-state of the system) as depicted by Fig.4(A). However, as indicated by Bogoliubov's theory[8], this does not hold for a SIB; $N_{p=0}$ gets depleted by $N_{p \neq 0}=N-N_{p=0}$ which move to different states of $p \neq 0$ beca use the G-state energy of the system increases by certain value (say, $\Delta E_{\mathrm{o}}$ ) due to inter-particle repulsion $V^{\mathrm{R}}\left(r_{i j}\right)$; the momentum distribution of particles $(N(p))$ in the G-state of a $\mathrm{SIB}$, so expected is shown in Fig.4(B). $N_{p \neq 0}$ and $\Delta E_{0}$, presumably, increase with the increasing strength of $V^{\mathrm{R}}\left(r_{i j}\right)$. CMT calculations reveal that $p=0$ condensate gets depleted to a value as low as $\approx 10 \%[25-28]$ in He-II and $\approx 60 \%$ or more in TDG[40]. However, recent theoretical studies of He-II and TDG consider that S-phase of a SIB also has intensive pair coherent condensate (PCC), -analogous to the Cooper condensate in a Fermi liquid with an attraction between the fermions. Having a slightly different view point Jeon et al[47(a)] and Hao[47(b)] try to revive an old consideration[48(a-c)] and try to conclude that the unique properties of the S-phase of a SIB are the consequences of the condensation of only Cooper type pairs of ${ }^{4} \mathrm{He}$-atoms. Interestingly, since $N(p)$ in the G-state of a SIB, as concluded 
by all these theories, does not differ qualitatively from that depicted in Fig.4(B), it is clear that these theories differ only for their presumed emphasis on single particle condensate or pair condensate or composite condensate as the origin of superfluidity and related aspects. However, in the absence of a clear experimental evidence in support of the existence of any form of the condensate, this difference may have only academic importance; what remains to be noted is that all these studies use SPB to describe a SIB and approximation methods (perturbative or variational) to reach their conclusions and in this context it is important to note that single particle representation does not agree with a physical reality of the LT states of a SIB in which particles bound to have states of their wave superposition which cannot be understood unless we take at least two waves as discussed in Section 2.2.

\subsection{Should Results of PPB and SPB Approaches Differ?}

"Physical quantities should not depend on the basis (SPB or PPB) used for describing a system, if the theoretical treatment is exact. This motivated us to examine the reason for the difference of G-state of a SIB concluded by SPB (Fig.4(B)) and that concluded by PPB (Fig.4(C)) because no system can be correctly understood unless we have the correct understanding of its G-state. In this context our simple mathematical analysis[79], unequivocally, concludes that $N(p)$ (Fig.4(B)) does not represent a state of minimum possible energy as expected for every physical system in its G-state. Subjecting $N(p)$ (Fig.4(B)) to the condition which can minimize its energy, we discovered[79] that: (i) all particles in the true G-state of a SIB should have identically equal energy $\varepsilon_{\mathrm{o}}=h^{2} / 8 m d^{2}$ and equivalent momentum $q=q_{\mathrm{o}}=$ $\pi / d$, and (ii) no particle has a $q<q_{\mathrm{o}}$ which establishes the absence of $p=0$ condensate. In the light of the fact that these inferences agree exactly with corresponding ones of our theory and our results (for the G-state) of a SIB can be obtained from those of CMT (not vice versa) simply by minimizing the energy of $N(p)$ (Fig.4(B)), we can use the opening statement of this section to state that our theory is nearly exact.

A quantum theory is said to be exact if it finds solutions of the Schrodinger equation of a system without leaving any part of the potential involved. To this effect it may be noted that our theory starts by using $V\left(r_{i j}\right)=V^{\mathrm{R}}\left(r_{i j}\right)+V^{\mathrm{A}}\left(r_{i j}\right)$ without any approximation. In a next step it uses an approximation $V^{\mathrm{R}}\left(r_{i j}\right) \equiv V_{\mathrm{HC}}\left(r_{i j}\right) \equiv \mathrm{A}\left(r_{i j}\right) \delta\left(r_{i j}\right)$ and replace the contribution of $V^{\mathrm{A}}\left(r_{i j}\right)$ by $-V_{\mathrm{o}}$ in a good agreement with the physical realities of a fluid (i.e., particles in a fluid move freely like impenetrable hard balls; two particles encounter infinitely strong potential only when their bodies have a mutual touch). Finally, when the system is dominated by the wave nature of particles at LTs with their wave superposition, our theory considers their zero-point repulsion (a combine of $\mathrm{A}(r) \delta(r)$ and WP manifestation of a particle) in its totality and derives its consequences by balancing $f_{\mathrm{o}}$ and $f_{\mathrm{a}}$ (Section 5.1) and in this process it uses only one approximation in which $f_{\mathrm{a}}$ is derived from a harmonic potential, $(\mathrm{c} / 2) x^{2}$, while its source potential $V^{\mathrm{A}}\left(r_{i j}\right)$ can contribute an-harmonic terms too. Evidently, all these points justify that our theory is nearly exact.

\section{Conclusions}

This paper reports a nearly exact microscopic theory of a SIB by solving $\mathrm{N}-$ body Schrodinger differential equation by using the simplest possible method. The accuracy of our theory finds strong experimental support from: (i) the agreement of its concluded excitation spectrum, $E(Q)=$ $\hbar^{2} Q^{2} / 4 m S(Q)[65,70]$, (planned to be published as Paper-II) with experimentally observed $E(Q)$ of LHe-4[25], (ii) conformity of its calculated values of the parameters of two fluid hydrodynamics $\left(v i z ., \rho_{\mathrm{s}}(T), \rho_{\mathrm{n}}(T)\right.$, etc. reported in[71]) with corresponding experimental results for He-II[5], (iii) experimentally observed quantized circulation, (iv) flow of $\rho_{\mathrm{s}}(T)$ in the direction opposite to that of $\rho_{\mathrm{n}}(T)$ which flows from the region of higher $T$ to that of lower $T$, etc.

Its concluded energy gap $\left(E_{\mathrm{g}}(T)\right)$ between the S-state and $\mathrm{N}$-state of LHe-4 not only serves as the origin of superfluidity and related aspects but also provides reasons for the stability of the S-state against the small energy perturbations. This is demonstrated by the fact that the critical velocity of its linear flow $\left(v_{\mathrm{c}}\right)$ and that of its rotational flow $\left(\Omega_{\mathrm{c}}\right)$ (for which its superfluidity is totally lost) obtained by us[71] from $E_{\mathrm{g}}(T)$ for He-II agree closely with experiments[5]. Since the maximum value of per particle share in $E_{\mathrm{g}}(T)$ (Eqn.(60)) for He-II falls around $\mathrm{mK}$ energy, superfluidity is an energetically weak effect and this is evident from the small values of $v_{\mathrm{c}}$ and $\Omega_{\mathrm{c}}$.

As concluded in Section 4.4, BEC of bosons (in a SNIB as well as a SIB like LHe-4) has a unified picture in the framework of our NCMT; for both cases it can be identified as a condensation of particles in their G-state where each particle has $\langle p\rangle=0$ (not $p=0$ ). It may be noted that: (i) momentum of a particle in both systems (SNIB and SIB) kept in finite $\mathrm{V}$ does not remain a good quantum number, particularly, when their $\lambda_{T}$ becomes of the order of $d$ and (ii) each particle even in the G-state of a SNIB has non-zero energy $\left(\approx h^{2} / 8 m \mathrm{~V}^{2 / 3}\right)$ and corresponding non-zero momentum $\left(q=q_{\mathrm{o}} \approx \pi / \mathrm{V}^{1 / 3}\right)$. The fact that corresponding energy and momentum values for a SIB fall at about $N^{2 / 3}$ and $N^{1 / 3}$ times higher speaks of the impact of the $\mathrm{HC}$ interaction among the particles in a SIB; $N=1000$, these factors, respectively fall at 100 and 10 , while for $N=10^{24}$, they fall at $10^{16}$ and $10^{8}$ which are very large factor. To this effect we note that a particle in a SNIB has no means to know about the presence of other particles hence each particle behaves as if the entire $\mathrm{V}$ belongs to it. However, this is not the case for a SIB, since effective volume available for each particle is only $\mathrm{V} / N$.

In what follows from $\rho_{\mathrm{s}}\left(T_{\lambda}\right)=0$, it can be stated that the onset of BEC at $T=T_{\lambda}$ occurs merely few particles occupying the state of $q=q_{\mathrm{o}}$ and $K=0$ and ends with all particles having this state at $T=0$. Evidently, the $<p>=0$ condensation in a SIB grows from $n_{<\mathrm{p}>=0}\left(T_{\lambda}\right)=0$ to reach $n_{\langle\mathrm{p}>=0}(T=0)=1.0(100 \%$ condensation). Since this is consistent with $\rho_{\mathrm{s}}\left(T_{\lambda}\right)=0$ and $\rho_{\mathrm{s}}(0)=1.0$, our NCMT has no difficulty (of the type faced by 
CMT where only $\approx 10 \%{ }^{4} \mathrm{He}$ atoms are found to have $p=0$ even at $T=0$ ) in using two fluid description of He-II.

CMT based on SPB approach can also help in concluding the true G-state (Fig.4(C)) of a SIB provided the energy (corresponding to the G-state $N(p)$ (Fig.4(B)) concluded by it) is minimized by making pairs of particles, -as shown in[79]. Evidently, the difference of CMT results with those of ours are the consequences of its SPB approach clubbed different possible presumptions and approximations, -not of the mathematical approach(es) used in it.

We applied our approach to study some other MBQS. As, discussed briefly in[80], it has great potential to unify the physics of widely different systems of interacting bosons and fermions including low dimensional systems, newly discovered BEC states of TDG, etc. Our studies of $N$ HC particles in 1-D box[81] and basic foundations of the microscopic theory of superconductivity[82] make it evident that a fermionic system differs from a bosonic system for its particle-distribution on the allowed states of $K$-motions which are constrained to follow Fermi-Dirac statistics in place of Bose-Einstein statistics followed for a bosonic system. Consequently, a fermionic system retains more energy in $K$-motions of particles even at $T=0$. Consequently, fermions do not assume stable bound pairs unless their $T$ falls below the $T$ equivalent of $E_{g}(T)$ where $q$-motions get delinked from $\mathrm{K}$-motions; note that binding of particles controls only $q$-motions (relative motions) not the $K$-motions. This not only explains how excess energy of $K$-motions pushes down the $T$ of the onset of superfluidity in a fermionic system in comparison to a bosonic system but also underlines the fact that superfluidity of both systems has a common origin which lies with the formation of bound SMW pairs. A SMW bound pair differs slightly from a Cooper pair for the basic source of its binding (Section 5.1(v)). Our approach finds that superfluid transition in a SIB should occur at a $T \approx T_{\mathrm{o}}\left(\equiv \varepsilon_{\mathrm{o}}\right)$, while that in a fermionic system should occur around a $T \equiv E_{\mathrm{g}}(0) / N$ and this agrees closely with experimental values[36] of the temperature of the onset of superfluidity in LHe-4 and LHe-3, respectively.

It is interesting to find that our theory of superconductivity[82] can account for the highest $T_{\mathrm{c}}$ of a high $T$ superconductor that we know to-day. It can also explain the pressure dependence of the $T_{\mathrm{c}}$ of superfluid transition in LHe-3 at quantitative scale[83]. The zero-point force of a particle emphasized in these studies as the origin of a strain in the structure of the neighboring particles (viz. the lattice in case of superconductors and inter-atomic bonds in liquid ${ }^{3} \mathrm{He}$ ) is a natural consequence of wave nature of particles for which a particle behaves as a WP and occupies exclusively a space of size $\lambda / 2$. This is clearly supported by the experimental observation that an electron in LHe-4 and LHe-3 exclusively occupies large size spherical cavity (or what is known as electron bubble) which has a radius of the order of $\approx 19 \AA \hat{\text { and }}$ the volume expansion exhibited by LHe-3 on its cooling below about $0.6 \mathrm{~K}$ [2(a)]. In a recent paper[84], we explained that only our theory concludes an ordered arrangement of particles (free from collisions) in the S-phase of a SIB de- sired to explain the experimental observation of Stark effect of roton transition [85] in He-II. Evidently, the observation of Stark effect renders strong experimental support to our theory and the absence of $p=0$ condensate.

Our non-conventional approach works well in developing the microscopic theory of a bosonic system like LHe-4 (as discussed in this paper) and a fermionic system like electron fluid in superconductors discussed in[82] (valid also for liquid ${ }^{3} \mathrm{He}$ type systems). We hope that a critical discussion and scientific debate on our approach may further improve its strength and accuracy and it will help in concluding the desired theory of widely different many body systems of interacting bosons and fermions.

It may be noted that our theory uses only the realistic picture of a fluid. It makes no assumption such as the existence of $p=0$ condensate as the origin of superfluidity used by CMT. It is, obviously, consistent with the well known philosophical principle, -the Occam's razor, which states that the explanation of a phenomenon should make as few assumptions as possible or the simplest solution of a problem is preferable to more complicated solutions. In this context it is interesting that our theory helps in clarifying the difference between S-fluid and N-fluid states even to a layman since these states can be identified, respectively, with: (i) the ordered positions and motions of soldiers in an organized army platoon and (ii) random positions and motions of people in a crowd. While the people in the former have coherent motion with no chance of mutual collision, those in the latter have incoherent motion with high probability of such collisions. The analogy seems to agree with what has been depicted in[86] about the BEC state of atoms in TDG and what has been perceived by Leggett[87] about ${ }^{4} \mathrm{He}$ atoms in the so called $p=0$ condensate in He-II but with a difference. According to our theory, all atoms in the S-state of a SIB behave like soldiers in an organized army platoon, while in view of $[86,87]$, this analogy applies only to the atoms which constitute the so called $p=0$ condensate (i.e. about $10 \%{ }^{4} \mathrm{He}$ atoms in He-II or about $60 \%$ atoms in BEC state of TDG).

In summary, we succeeded in developing the long awaited microscopic theory of a SIB like LHe-4 and TDGs. It finds that superfluidity of a SIB is basically a property of its G-state configuration which persists at all $T<T_{\lambda}$; it can be exhibited by a SIB of any size (as small as a microscopic cluster having a few bosons or as large as bulk LHe-4) provided the system retains fluidity at $T_{\mathrm{o}}\left(\equiv \varepsilon_{\mathrm{o}}\right)$. The theory predicts that such a SIB would exhibit-ve thermal expansion coefficient around its transition to superfluid state. It also predicts the presence of phonon like waves of oscillations in $q$ values around $q_{\mathrm{o}}$ (proposed to be known as omon) which propagate from the points (or regions) of lower $T$ to those of higher $T$. For the first time our theory underlines the importance of the WP manifestation of a quantum particle and zero point force $f_{0}$ exerted by each of them on the neighboring particles in organising their relative positions in $r$ - and $\varphi$-spaces. Our similar study of $N$ interacting bosons confined to a 1-D box[81] clearly concludes that superfluidity and related properties can also be exhibited by systems of 
low dimensions (viz., 1-D and 2-D) below certain $T$ (of the order of $T_{\mathrm{o}}$ ). The results of our theory can have meeting points with those of CMT $[8,50]$ provided the energy corresponding to their concluded $N(p)$ (Fig.4(B) is minimized. This point is concluded in our recent paper[79(c)] which not only reveals the absence of $p=0$ condensate but also demonstrates the possibility of $N(=2,3, \ldots)$ body correlations in superfluid state of a SIB; these correlations have been central to the recent developments in CMT[43-46]. The fact that our theory has been developed by solving its $N$-body Schrodinger equation in its standard differential form breaks the myth that such a solution is practically impossible to achieve. It also sets aside the speculation[5] that wave mechanics does not have basic principles to explain superfluidity of He-II. We hope that this theory would be accepted widely by the physics community and in this context, we would like to submit that 75 years is a long time to test the validity of the idea of $p=0$ condensate as the origin of superfluidity. It is high time to give a chance to newer ideas.

\section{ACKNOWLEDGEMENTS}

Acknowledgement: The author has no words to express his gratitude to Prof. A. J. Leggett for his valuable comments. $\mathrm{He}$ is thankful to N.M. Blagoveshchenskii, Bimalendu Dey, Jorge E. Hirsch, Ashok Kumar, and B. K. Srivastava, for their encouraging communication, and his students D.R. Chaudhury, S. Chutia, L. Chhangte, Ningthoujam T. Chanu, and S. Dey for their efforts to take this theory forward.

\section{Appendix - A}

\section{A Critical Analysis of $<\mathbf{A} \boldsymbol{\delta}(\mathbf{x})>=\mathbf{0}$}

In general $\mathrm{A}(x)$, in $V_{\mathrm{HC}}(x) \equiv \mathrm{A}(x) \delta(x)$, can be such that $\mathrm{A}(x)$ $\rightarrow \infty$ for $x \rightarrow 0$. It can, therefore, be expressed as

$$
\mathrm{A}(x)=\mathrm{B} x^{-(1+\alpha)}
$$

with B and $\alpha$ being $>0$. Using $\Psi(x, X)^{ \pm}=\sqrt{ } 2 \psi_{\mathrm{k}}(x)^{ \pm} \exp (\mathrm{i} K X)$ as the 1-D analogue of the pair states $\Psi(r, R)^{ \pm}$(Eqn.10) with $\psi_{\mathrm{k}}(x)^{-}=\sin (k x / 2)$ in place of $\left.\psi_{\mathrm{k}}(r)^{-}=\sin (\mathbf{k} . \mathbf{r}) / 2\right)($ Eqn.11) and $\varphi_{\mathrm{k}}(x)^{+}=\sin (|k x / 2|)$ in place of $\left.\varphi_{\mathrm{k}}(r)^{+}=\sin (|\mathbf{k} . \mathbf{r}|) / 2\right)$ (Eqn.12). Since $\left|\psi_{\mathrm{k}}(x)^{-}\right|^{2}=\left|\varphi_{\mathrm{k}}(x)^{+}\right|^{2}$, we find that

$$
<\mathrm{A}(x) \delta(x)>=\mathrm{B}\left\{\left[2 \sin ^{2}(k x / 2) / x^{(1+\alpha)}\right.\right.
$$

is an in-determinant which can be simplified to $\mathrm{B} k^{2} x^{1-\alpha} / 2$ for $x \approx 0$. Evidently, when $x \rightarrow 0$ we have

a finite $+v e$ value

$$
<\mathrm{A}(x) \delta(x)>=0 \text { for } \alpha<1
$$

$$
<\mathrm{A}(x) \delta(x)>=\mathrm{B} k^{2} / 2 \quad \text { for } \alpha=1
$$

and

$$
<\mathrm{A}(x) \delta(x)>=\infty \text { for } \alpha>1
$$

Since no physical system can ever occupy a state of $\infty$ potential energy, $\alpha>1$ corresponds to a physically uninteresting case. Among the remaining $\alpha$ values (corresponding to physically interesting configurations), $\alpha=1$ is the sole point on the $\alpha$-line for which $<\mathrm{A}(x) \delta(x)>\neq 0$. It stands as a sharp divide between $<\mathrm{A}(x) \delta(x)>=0$ (for $\alpha<1$ ) and $<\mathrm{A}(x) \delta(x)>=$ $\infty$ (for $\alpha>1$ ). To understand the physical significance of these results, we note the following.

1. $<\mathrm{A}(x) \delta(x)>=0$ is clearly valid for $\alpha<1$

2. Using Eqn.(A-4) and $h_{\mathrm{i}}=-\left(\hbar^{2} / 2 m\right) \partial x_{\mathrm{i}}[1-\mathrm{D}$ analogue of 3-D $h_{\mathrm{i}}$ (Eqn.1)], we evaluate $E(2)=<H(2)>=<h_{1}+h_{2}+\mathrm{A}(x)$ $\delta(x)>$ for $\Psi(x, X)^{ \pm}$state and find

$$
E_{r e l}(2)=\hbar^{2} k^{2} / 4 m+\mathrm{B} k^{2} / 2=\hbar^{2} k^{2} / 4 m\left(1+2 \mathrm{~B} m / \hbar^{2}\right)
$$

which represents the total energy expectation of the relative motion of two $\mathrm{HC}$ particles for $\alpha=1$ case. We note that $<\mathrm{A}(x) \delta(x)>=\mathrm{B} k^{2} / 2$ and $\hbar^{2} k^{2} / 4 m$ can be absorbed into one single term by defining

$$
E_{r e l}(2)^{*}=\hbar^{2} k^{2} / 4 m^{*} \text { with } m^{*}=m /\left(1+2 \mathrm{~B} m / \hbar^{2}\right) \quad \text { (A-7) }
$$

which justifies $\langle\mathrm{A}(x) \delta(x)>=0$ also for $\alpha=1$ if $m$ is replaced by $m^{*}$. Although, this shows that Eqn.(14) stands valid for all situations of physical interest, however, it does not explain why the energy of the relative motion of two $\mathrm{HC}$ particles at $x \gg>0$ should be $E_{r e l}(2)^{*}$, rather than $E_{\mathrm{k}}=\mathrm{h}^{2} k^{2} / 4 m$ expected for the fact that $\mathrm{A}(x) \delta(x)$ is experienced only if $x=0$, and why $<\mathrm{A}(x) \delta(x)>\left(\right.$ as indicated by its value $\mathrm{B} k^{2} / 2$ ) should be kinetic in nature; note that $\langle\mathrm{A}(x) \delta(x)\rangle=\mathrm{B} k^{2} / 2$ does not have potential energy character of $\mathrm{A}(x) \delta(x)$ because it is neither a function of $x$ nor of $\langle x\rangle$. Evidently, $\langle\mathrm{A}(x) \delta(x)\rangle=$ $\mathrm{B} k^{2} / 2$ needs an alternative explanation (cf. points 3 and 4 , below).

3. Writing $\left\langle\mathrm{A}(x) \delta(x)>=\int_{0} \epsilon \ldots+\int_{\epsilon}^{\infty} \ldots\right.$ and evaluating these integrals under the limit $0 \rightarrow \epsilon$ and $\epsilon \rightarrow 0$, we find that $<\mathrm{A}(x) \delta(x)>=\mathrm{B} k^{2} / 2$ (Eqn.A-4) is the solitary contribution from $x=0$ only. Similarly, we also observe that $\left.<-\hbar^{2} / m\right) \partial x^{2}>=\hbar^{2} k^{2} / 4 m$ (the expectation value of kinetic energy of relative motion) has no contribution from $x=0$; the related integral is found to be independent of the inclusion or exclusion of $x=0$ point. These observations prove that Eqn.A-4 is a result of the conservation of energy of the pair $\left.<-\hbar^{2} / m\right) \partial x^{2}>$. It appears that two particles in their relative motion have only kinetic energy $\left(E_{\mathrm{k}}=\hbar^{2} k^{2} / 4 m\right)$, till they reach the point of their collision at $x=0$ where they come to a halt and $\hbar^{2} k^{2} / 4 m$ gets transformed into an equal amount of potential energy (as a result of energy conservation). This potential energy should, naturally, be proportional to $k^{2}$ as seen in Eqn.A-4. This not only implies that B $k^{2} / 2$ in Eqn.A-4 does not represent an additional energy to be added in $\left.<-\hbar^{2} / m\right) \partial x^{2}>=\hbar^{2} k^{2} / 4 m$ to determine $E_{r e l}(2)$ as appears from Eqn.(A-6) but also suggests that the physical meaning of non-zero $<\mathrm{A}(x) \delta(x)>$ of an ill behaved potential such as $\mathrm{A}(x) \delta(x)$ should be distinguished from that of $\langle V(x)\rangle$ of a well behaved (i.e. continuous and differentiable) $V(x)$. Using the said conservation of $\hbar^{2} k^{2} / 4 m$ energy at $x=0$, we can equate it to $\mathrm{B} k^{2} / 2$ and find that

$$
\mathrm{B}=\hbar^{2} / 2 m \text { and } V(x)=\mathrm{A}(x) \delta(x)=\hbar^{2} / 2 m x^{-(1+\alpha)} \delta(x)(\mathrm{A}-8)
$$

In wave mechanics, two colliding particles either exchange their positions (across the point $x=0$ ) or their momenta. In the former case they can be seen to cross through their $\delta$-potential, possibly, by some kind of tunneling (in which their kinetic energy does not transform into potential energy), while in the latter case they return back on their path after a halt at $\mathrm{x}=0$ in which case their potential energy rises at the cost of their kinetic energy which reappears as the two particles move away from $x=0$ point. While, the two pos- 
sibilities can be identified, respectively, with Eqn.A-3 and Eqn.A-4 of this Appendix, however, it is also clear that one has no means to decide whether the two particles exchanged their positions or their momenta which implies that the two situations are indistinguishable and it should not be surprising if $<\mathrm{A}(x) \delta(\mathrm{x})>$ is found to have any value between 0 to $\mathrm{B} k^{2} / 2$ (i.e. $<\mathrm{A}(x) \delta(x)>$ has an uncertainty as large as $\mathrm{B} k^{2} / 2$ ); this is particularly possible because a collision of two HC particles at $x=0$ (i.e. an exact $x$ ) is a state of zero uncertainty in $x$ and infinitely high uncertainty in $k$ or $E_{\mathrm{k}}=\hbar^{2} k^{2} / 4 m$.

In summary, non-zero $<\mathrm{A}(x) \delta(x)>=\mathrm{B} k^{2} / 2$, observed for $\alpha=1$, should be treated as fictitious. Else it can best be attributed to energy conservation at $\mathrm{x}=0$ which implies that $<\mathrm{A}(x) \delta(x)>=0$ (i.e. Eqn.14) is relevant for all possible physical situations of two HC particles that can be represented by $\alpha \leq 1$.

\section{REFERENCES}

[1] (a) P. Kapitza, Nature 141 (1938), 74-74, (b) J.F. Allen and A.P. Misener, Nature 141 (1938), 75-75.

[2] (a) J. Wilks, The properties of Liquid and Solid Helium. Clarendon Press, Oxford (1967).(b) J. Wilks and D.S. Betts, An Introduction to Liquid Helium. Clarendon Press, Oxford (1987).

[3] Z.M. Galasiewics, Helium 4. Pergamon, Oxford (1971).

[4] A.D.B. Woods and R.A. Cowley, Rep. Prog. Phys. 36 (1973), 1135-1231.

[5] S.J. Putterman, Superfluid Hydrodynamics North -Holland, Amsterdam (1974).

[6] (a) L.D. Landau, J. Phys. (USSR) 5 (1941), 71; Reprinted in English in Ref.[3] pp 191-233, (b) L.D. Landau, J. Phys. (USSR) 11 (1947), 91; Reprinted in English in Ref.[3] pp 243-246.

[7] (1) Superfluid of viscosity $\eta=0$ and entropy $S=0$ and (2) Normal fluid of $\eta=0$ and $S=0$.

[8] N.N. Bogoliubov, J, Phys. (USSR) 11 (1947), 23; Reprinted in English in Ref.[3], pp 247-267.

[9] R.K. Pathria, Statistical Mechanics, Pergamon Press Oxford (1976).

[10] In what follows from the theory of Bose Einstein condensation[9], a system of non-interacting bosons (SNIB) has an onset of $p=0$ condensate, -a macroscopic number of particles $\left(N_{p=0}\right)$ occupying single particle state of $p=0$ at certain $T=$ $T_{\mathrm{BEC}}$ on cooling the system. $N_{p=0}(T)$ rises smoothly from $N_{p=0}\left(T_{\mathrm{BEC}}\right)=0$ to $N_{p=0}(0)=N$. According to Bogoliubov[8], hard core repulsion depletes $N_{p=0}(T)$ by pushing $\mathrm{N}-N_{p=0}(T)$ particles in higher energy states of $\mathrm{p} \neq 0$.

[11] F. London, Nature 141 (1938), 643-644; Phys. Rev. 54 (1938), 947-954; Superfluid, Wiley, New York (1950).

[12] R.P. Feynman, Phys. Rev. 91 (1953), 1291-1301.

[13] R.P. Feynman, Phys. Rev. 91 (1953), 1301-1308.
[14] R.P. Feynman, Phys. Rev. 94 (1954), 262-277.

[15] R.P. Feynman, Applications of Quantum Mechanics to Liquid Helium, Progress in Low Temperature Physics (C.J. Gorter, editor). 1 (1954),17-53, North-Holland, Amsterdam, Chapter II.

[16] M. Cohen and R.P. Feynman, Phys. Rev 102 (1956), 1189-1204.

[17] M. Cohen and R.P. Feynman, Phys. Rev 107 (1957), 13-24.

[18] A. Fetter and J. Walecka, Quantum Theory of Many Particle System McGraw-Hill, New York (1971).

[19] E. Feenberg, Theory of Quantum Fluids Academic Press, New York (1969).

[20] J.G.M. Armitage and I.E. Ferquhar, (eds.): The Helium Liquids. Academic Press, London (1975).

[21] K.H. Benneman and J.B. Ketterson, (eds.): The Physics of Liquid and Solid Helium, Part-I. Wiley, New York (1976).

[22] C. W. Woo in Ref.[21], pp 349-501

[23] N. Toyoda, Ann. Phys.(NY) 141 (1982), 154-178.

[24] D.M. Ceperley, Rev. Mod. Phys. 67 (1995), 279-355.

[25] H.R. Glyde and E.C. Svensson, Solid and liquid helium. In D.L. Rice and K. Skold (eds.) Methods of Experimental Physics-Neutron Scattering, vol. 23, Part B, pp 303-403. Academic, San Diego (1987).

[26] R.N. Silver and P.E. Sokol (eds.), Momentum Distribution. Plenum Press, New York (1989).

[27] R.N. Silver, Superfluid helium and neutron scattering, a new chapter in the condensate saga, in Los Almos Science (No. 19), N.G. Cooper (ed.) (1990), pp 158-179.

[28] P.E. Sokol in Bose Einstein Condensation, A. Griffin, D.W. Snoke and A. Stringari (eds.), Cambridge University Press, Cambridge, (1996), pp 51-85.

[29] C.H. Aldrich and D. Pines, J. Low Temp. Phys. 25 (1979), 677-690.

[30] J.A. Geurst, Physica 153 (1988), 166-180.

[31] A. Griffin, Excitations in a Bose condensed liquid, Cambridge University Press, Cambridge, (1993).

[32] R.J. Donnelly, Quantized Vortices in Helium II, Cambridge University Press (1991).

[33] C.F. Barenghi, R.J. Donnelly and W.F. Vinnen (eds.), Quantized Vortex Dynamics and Superfluid Turbulence, Springer Verlag, Berlin (2001).

[34] D.R. Tilley and J. Tilley, Superfluidity and Superconductivity, Adam Hilger, Bristol (1990).

[35] T. Guenault, Basic Superfluids, CRC Press (2002).

[36] C. Enss and S. Hunklinger, Low Temperature Physics, Springer Verlag, Berlin, (2005).

[37] (a) V.L. Ginzburg and L.P. Pitaevskii, On the theory of superfluidity, Zh. Eksp. Teor. Fiz. 34 ((1958), 1240[Sov. Phys. JETP 7 (1958), 858], (b) V.L. Ginzburg and A.A. Sobyanin, Superfluidity of helium II near the $\lambda$-point, in Superconduc- 
tivity, Superdiamagnetism, Superfluidity, V.L. Ginzburg (ed.), p. 242, Mir, Moscow, (1987). (c) V.L. Ginzburg, and A.A. Sobyanin, Superfluidity of helium II near the $\lambda$-point, Usp. Fiz. Nauk 154 (1988), 545[Sov. Phys. Usp. 31 (1988), 289]; Japan J. Appl. Phys. 26 (Suppl.26-3) (1987), 1785. (d) V.L. Ginzburg, Rev. Mod. Phys. 76 (2004), 981-998.

[38] For example He-II at $T=0$ has $10 \%{ }^{4} \mathrm{He}$-atoms as $p=0$ condensate and $90 \%$ atoms as non-condensate which clearly does not have $\mathrm{S}=0$. Hence it not clear how such a He-II can conform to $S=0$ of superfluid component with $\rho_{s}(0)=\rho$. Similarly, it is also not clear how particles, having different $\lambda$ due to their different momenta, exhibit coherence of their motion.

[39] A.J. Leggett, Rev. Mod. Phys. 71 (1999), S318-S323; an important remark by Prof. Leggett about the existence of $p=0$ condensate in He-II is worth quoting. He states, "In the sixty years since London's original proposal, while there has been almost universal belief that the key to superfluidity is indeed the onset of BEC at $T_{\lambda}$ it has proved very difficult, if not impossible, to verify the existence of the latter phenomenon directly. The main evidence for it comes from high energy neutron scattering and, very recently, from the spectrum of atoms evaporated from the liquid surface and while both are certainly consistent with the existence of a condensate fraction of approximately $10 \%$, neither can be said to establish it beyond all possible doubts."

[40] (a) F. Dalfovo, S. Giorgini, L.P. Pitaevskii, and S. Stringari, Rev. Mod. Phys. 71 (1999), 463-512, and Arxiv/Cond -mat/9806038. (b) A. Fetter, Rev. Mod. Phys., 81 (2009), 647.

[41] C.J. Pethick and H. Smith, Bose-Einstein Condensation in Dilute Gases, Cambridge University Press, 2002.

[42] L. Pitaevskii and S. Stringari, Bose-Einstein Condensation. Oxford Science Publications, 2002.

[43] (a) V.A. Zagrebnov and J.-B. Bru, Phys. Rep. 350 (2001), 291-434, (b) S. Adams and J, -B. Bru, Physica A 332 (2003), $60-78$, (c) J.V. Pule, A.F. Verbeure and V.A. Zagrebnov, On Solvable Boson Models : www.ma.utexas. edu /mp arc/c/08/08-13.pdf (2007).

[44] A.S. Peletminskii and S.V. Peletminskii, Low Temp. Phys. 36 (2010), 693-699.

[45] (a) V.I. Yukalov, and H. Kleinert, Phys. Rev. A73 (2006), 063612, (b) V.I. Yukalov, Annals Phys. 323 (2008), 461-499, (c) V.I. Yukalov, Phys. Part. Nucl. 42 (2011), 460-513.

[46] (a) E.A. Pashitskii, Low Temp. Phys. 25 (1999), 81-99, (b) E.A. Pashitskii and S.I. Vil'chinskii, Low Temp. Phys. 27 (2001), 185-195, (c) E.A. Pashitskii, S.V. Mashkevich and S.I. Vil'chinskii, Phys. Rev. Lett, 89 (2002), 075301, (d) E.A. Pashitskii, S. I. Vilchinski and A.V. Chumachenko, Low Temp. Phys. 36 (2010), 576-581.

[47] (a) G.S. Jeon, L. Yin, S.W. Rhee and D.J. Thouless, Phys. Rev. A 66 (2002), 011603(R), (b) Z. Hao, Theory for superfluidity in a Bose system arxiv:Cond-mat/0807.1503v2

[48] (a) J.G. Valatin, and D. Butler, Nuovo Cimento 10 (1958), 37, (b) W.A.B. Evans and Y. Imry, Nuovo Cimento B 63 (1969), 155, (c) P. Nozieres and D.S. James, J. Physique 43 (1982), 1133.

[49] H. Shi and A. Griffin, Phys. Rep. 304 (1998), 1-87.

[50] J.O. Anderson, Rev. Mod. Phys. 76, 599-639 (2004).
[51] M. D. Tomchenko, Assian J. Phys. 18 (2009), 245-254; Uneven horizon or several words about the superfluid ${ }^{4} \mathrm{He}$ theory, arXiv:0904.4434.

[52] K. Huang, Statistical Mechanics, Wiley Eastern Limited, New Delhi (1991), Chapt 13.

[53] Y.S. Jain, Cent. Euro J. Phys. 2 (2004), 709-719; a small typographic error in this paper is corrected in its version placed in the archive (arxiv.org/quant-ph/0603233).

[54] Since the excitation wave length $(\Lambda)$ of particles having $\varepsilon \geq \varepsilon_{\mathrm{c}}$ satisfies $\Lambda / 2 \leq \sigma$ and two particles cannot have $r<\sigma$ for their $\mathrm{HC}$ nature, it is clear that particles with $\Lambda / 2<\sigma$ cannot have positions in agreement with Eqn.29. This means that such particles do not have the correlations of their positions in $r$, $k$ - and $\varphi$-spaces.

[55] O. Penrose and L. Onsager, Phys. Rev. 104 (1956), 576-584.

[56] C.N. Yang, Rev. Mod. Phys. 34 ((1962), 694-704.

[57] J. Goldstone, N. Cim. 19 (1961), 154-164.

[58] P.W. Anderson, Rev. Mod. Phys. 38 (1966), 298-310.

[59] G. Taubes, Science 269 (1995), 152-153.

[60] Y.S. Jain, Physical behavior of a system representing a particle in a box having flexible size : arXiv/condmat.matrl.sci:0807.0732v2.Ref.[9], p 134.

[61] V.G. Levich, Theoretical Physics Vol. 4, North-Holland, Amsterdam (1973), pp 12-14.

[62] G.E. Uhlenbeck and L. Gropper, Phys. Rev. 41 (1932), 79-90.

[63] M. Karplus and R.N. Porter, Atoms and Molecules, The Benjamin/Cummins, Menlo Park (1970) Chapter 6.

[64] Y.S. Jain, Macro-orbitals and microscopic theory of a system of interacting bosons,arXiv:cond-mat/0606571.

[65] We note that per particle binding energy $E_{\mathrm{g}}(T) / N$ (Eqn. 56) (which is expected to be of the order of $|\beta(T)|$ ) is $<<k_{B} T_{\mathrm{o}}$ since $\Delta d / d<<d$. However, the binding $\left(E_{\mathrm{b}}\right)$ of particles in $\xi(T)^{3}$ volume[with $\xi(T)$ (Eqn.60) being the coherence length], which have coherence of their motion, is estimated to be $E_{\mathrm{b}}=-\left(\xi(T)^{3} / d^{3}\right)\left(E_{\mathrm{g}}(T) / N\right) \approx-k_{B} T_{\mathrm{o}}(\xi(T) / d)^{3}(\Delta d) / d$ which is clearly $>k_{B} T_{\lambda}$ since $\xi$ for LHe-4 is found to vary from $\approx$ 102.d at $T=0$ to $\infty$ at $T_{\lambda}$.

[66] C.J. Foot and A.M. Steane, Nature 376 (1995), 213-214.

[67] J. Bardeen, L.N. Cooper and J.R. Schrieffer, Phys. Rev. 108 (1957), 1175-1204.

[68] R.P. Feynman, Statistical Mechanics, Benjamin (1976).

[69] Y.S. Jain, A Study of Elementary Excitations of Liquid Helium-4 Using Macro-Orbital Microscopic Theory, arXiv:Cond-mat/0609418v1 (2006).

[70] (a) S. Chutia, A study of Certain Properties of Superfluid Helium-4 Using Macro-Orbital Theory, Ph.D. Dissertation, North-Eastern Hill University, Shillong (India),(2007),(b) Y.S. Jain and S. Chutia (unpublished work).

[71] S. Sachdev, Rev. Mod. Phys. 75 (2003), 913-932.

[72] (a) M. Rosenblit and J. Jortner, Phys. Rev. Lett. 75 (1995), 4079-4082; (b) M. Farnik, U. Henne, B. Samelin, and J.P. Toennies, Phys. Rev. Lett. 81 (1998), 3892-3895. (b) H. 
Marris and S. Balibar, Phys. To-day 53 (2000), 29-34.

[73] P. Hautojarvi, M.T. Loponen and K. Rytsola, J. Phys. Atom. Mole. Phys. 9 (1976), 411-422.

[74] J.P. Toennies and A.F. Vilesov, Ann. Rev. Phys. Chem. 49 (1998), 1-41.

[75] (a) S. Flugge, Practicle Quantum Mechanics, Springer-Verlag, (1974). (b) S. Dey and Y.S. Jain, On the wave mechanics of a particle in two different impenetrable spherical cavities, arXiv /quant-ph /1002.4308

[76] (a) S. Grebner, J.P. Toennies and A.F. Vilesov, Science 279 (1998), 2083-2086, (b) A.R.W. Mckellar, Y. Xu and W. Jager, Phys. Rev. Lett. 97 (2006), 183401, (c) K. Nauta and R.E. Miller, J. Chem. Phys. 115 (2001), 10254-10260, (d) A.R.W. Mckellar, J. Chem. Phys. 127 (2007), 044315.

[77] P. Kleban, Phys. Lett.A 49 (1974), 19-20.

[78] (a) Y.S. Jain, The $\mathrm{p}=0$ condensate is a myth, arXiv:cond-mat/1008.240V2 (2010); the basic foundation and important conclusions of paper-(a) are briefly discussed in[84]. (b) Y.S. Jain, Laws of nature forbid the existence of $p=0$ condensate in a system of interacting boson (unpublished).

[79] Y.S. Jain, Ind. J. Phys. 79 (2005), 1009-14; the factor $\mid \sin$ (k.r/2)| in Eqn.5 for $\Psi$ in this paper should be read as $\sin$ $|(\mathbf{k} . \mathbf{r} / 2)|$ and $E_{\mathrm{g}}(T) / N k_{B} T$ in Eqn.25 should be read as $E_{\mathrm{g}}(T) / N k_{B}$.

[80] Y.S. Jain, Ground state of a system of N hard core quantum particles in 1-D box, arXiv:cond-mat/0606409 (2006).

[81] Y.S. Jain, Basic foundations of the microscopic theory of super conductivity,arXiv:cond-mat/0603784, (2006).

[82] Y.S. Jain, Superfluid $T_{\mathrm{c}}$ of helium-3 and its pressure dependence. arXiv:cond- mat/0611298, (2006).

[83] Y.S. Jain, L. Chhangte, S. Chutia and S. Dey, Current Science, 101 (2011), 769-775.[Note: This paper also lists some other experimental observations which support the absence of $\mathrm{p}=0$ condensate and support CPA-WP of 4He-atoms in He-II].

[84] A. Rybalko, S.P. Rubets, E. Rudavskii, V. Tikhiy, S. Tarapov, R. Golovashchenko and V. Derkach, Phys. Rev. B76 (2007), 140503R.

[85] A picture depicted on the cover of Science magazine of December 22, 1995, declaring BEC in TDG as the molecule of the year.

[86] A Leggett, International. J. Mod. Phys. B, 15 (2001), 1312-1313. 